\title{
ALCUNE AGGIUNTE AL DIZIONARIO DI JOAN COROMINAS
}

Mi riferisco ai primi due tomi finora usciti del Diccionario critico etimológico de la lengua castellana, por J. Corominas, volumen I, A-C, volumen II, CH-K, Editorial Francke, Berna, Copyright I954 (ediz. spagnola, Gredos, Madrid). L'autore suggerisce la sigla DCEC, ma si usa citare semplicemente Corominas.

Quel che prima colpisce di questo lavoro è la sua impronta personalissima, lo spirito unitario e sintetico della diretta stesura, compiuta nel giro relativamente breve ed intenso di un quinquennio (I947-I95I). Como si approfondisce la nostra lettura, questo vivo elemento, direi, drammatico di composizione si rileva nella variabile tensione esterna ed interna tra etimologia e storia linguistica secondo un interesse proprio di essa storia, cioè, le origini e la fortuna del vocabolo-alfabeticamente costretto-nelle diverse correnti di cultura e di civiltà. Il carattere storico, pertanto, contro le stesse dichiarazioni del lessicografo, è preminente e qualificante; la parte migliore del dizionario si definisce là dove tutti gli strumenti della ricerca (selezione di glossari e repertori, filologia e critica testuale, comparazione e geografia linguistica, fonetica e sintassi, proprietà e tropismo, simbolismo espressivo e grammaticalizzazioni, cronache della moda e del costume, ecc.) convergono verso una puntuale storia semantica della lingua spagnola nel quadro generale della civiltà romanica. Si guardi 
a voci come cantiga e estribote, svolte in tutta la loro complessità di generi metricoletterari (però manca jarğ $a$ e altri termini affini, trascritti di recente, come aqra, dubait, haiki, ecc); o gorra, galocha nell'ambito della storia della moda; o hazaña, le cui difficoltà etimologiche tra etimo arabo e contaminazione castigliana si illustrano nella diacronia semantica da 'acción meritoria' a 'patraña', clalla cristallizazione giuridica all'uso volgare:

Tale storicismo, d'altra parte, non d viziato di atomismo o, peggio, di ideologismo preconcetto. La unità dei campi semantici è assicurata dalla ricerca instrinseca di ciascun vocabolo con i suoi derivati e composti in una fitta rete di opportuni riferimenti, che è la vita dell'etimo nella sua diacronia e nelle sue irradiazioni sistemiche e affinità estrasistemiche. I centri ordinarori sono scelti, non su astratti etimi preistorici, ma sulle figure iniziali e protostoriche del romanzo castigliano, nonché, sovente, sulla storia diversa, fonetica e semantica, di alcuni esiti: cosi, si distinguono cantar e cantiga; caldo, caliente e escalfar; casi e cachi-, dueño e ducnde, garbo e calibre; corazón, acordar e coro $I I I$; si vedano i chiarimenti alle pp. XIV-XV del Prefacio e, in particolare, $i$ veri e propri criteri del usentimiento lingüístico romance», della "solidariedad tra famiglie di voci, dell'affinità (talora meramente semantica) nella distribuzione dei derivati e composti, allotropi e omonimi. Ma solo una lunga consultazione darà modo di apprezzare l'ingegnosità del personale compromesso tra verità linguistica e adattamento alfabetico.

Ancora nel Prefacio Corominas giustamente pone in rilievo l'originalità e la cura degli spogli compituti nel campo delle lingue tecniche e specializzate (ad es., la botanica e la scienza nautica, i giochi e la malavita, il giture e le arti figurative), ma più comprensiva e significativa sembra essere la considerazione formale dei testi letterari, pur restando intesa la letteratura nella sua generale accezione di documento e fonte affidati alla scrittura. I a certezza filologica della citazione resta al servizio della più grande "amplitud semántica» della investigazione, la quale individua l'origine e la storia delle 
parole nel raggio di una qualificazione stilistica di carattere istituzionale, ma, non di rado, ove occorra, individuale e letterario stricto sensu (si annunzia alla fine dell'opera un indice degli emendamenti testuali!): a... cada vez más nos damos cuenta hoy en día de la importancia etimológica del punto de vista estilístico, de la pertenencia de una palabra a tal o cual ambiente social (germanía, etc.) o literario (léxico poético y análogos), o a tal o cual tecnicismo de oficio, y todo esto sólo podemos descubrirlo con un buen numero de citas literarias" (p. XXII).

Il dizionario di Corominas, infine, nel momento in cui definisce la lingua spagnola nel suo proptio àmbito storico $\mathrm{e}$ geografico, contribuisce poderosamente allo studio di tutte le lingua romanze e preromane, cioè del tesoro lessicale della civiltà occidentale; questa finalità è garantita dal metodo esaustivo col quale l'etimologia è perseguita nel campo misto delle lingue di contatto (arabo, basco, celtico, latino tardo, greco, germanico, americano aborigeno), e a maggior ragione nell'intera area interromanica. Attraverso gli indici finali ci sarà dato consultare un dizionario etimologico di tutte le lingue romanze iberiche, programmaticamente completo per il portoghese e il catalano.

La nostra recensione e il breve contributo lessicale rappresentano appena un segno modesto di riconoscenza per il ricevuto ausilio di un'opera di consultazione cosí vasta e ricca, e soprattutto sollecitante in ogni sua parte, strumento di lavoro indispensabile ad ogni letterato ispanista. Mi sono limitato a confrontare il dizionario con parziali spogli di alcuni testi letterari (Calila, Berceo, Cancioneros, Encina, F. de Herrera, Fray Luis de León), eseguiti all'occasione di corsi e seminari universitari. Mi sono accorto che non sempre il lessicografo attinge direttamente ai testi, specie se trattasi di opere di alto livello artistico; è il caso di Encina e, soprattutto, di Herrera. Il cantore di Luz è il grande intermediario tra umanesimo della corte di Juan II e barrocco, al cui limite si protende il suo maturo rinascimento nella prosa scientificoletteraria (anche le operette storiche andrebbero consultate). 
Corominas è attento alla corrente cultista, ma la clocumentazione non è sempre puntuale e precisa; sovente documenta nuove voci dotte in Lope o Quevedo o Cervantes, e a noi será facile retrodatarle a Herrera, il quale rimanda non di rado a Juan de Mena e anticipa Góngora; è un filone naturale e storico che si è perduto nell'economia del lavoro per una più urgente pressione in favore della fonetica e analogia romaniche e a scapito delle correnti umanistiche e dotte, pur se in questa direzione Corominas segni un enorme progresso rispetto a tutti gli altri dizionari storici. Un bel giorno occorrerà decidersi a compilare un vocabolario specializzato dei cultismi spagnoli, non mancando ormai una certa base monografica.

S'è accennato alla prosa scientifico-letteraria; e una docımentazione estratta dalle herreriane Anotaciones a Garcilaso, di cui daremo vari esempi, avrebbe rimediato ad analoga lacuna per quanto concerne la fondazione e fissazione in Spagna della terminologia grammaticale (fonetica, ortologia, metrica, rettorica, poetica, generi letterari, ecc.), oltre a molte voci delle scienze fisiche e waturali che Herrera traduce dagli antichi e dagli italiani; si tratta di una doviziosa enciclopedia del tardo rinascimento, sortita dall'aristotelismo laico e riformista e coeva del primo classicismo francese, la quale attende di essere immessa nella ricogniziones storica della lingua spagnola. Insomma, nelle Anotaciones le letterature egemoniche si castiglianizzano sul diretto spartito della lettera testuale, e già altrove (Sonetti di $F$. de Herrera con un saggio sulle Opere ed edizioni, in L'Albero, Lucugnano, I,ecce, luglio I955, estratto) ho ordinato i poeti latini, greci, umanisti e italiani da Herrera tradotti in detta opera; sono le fonti della lingua poetica herreriana scrutate e riprese dietro il primo italianismo garcilasiano.

Quanto alla terminologia scientifica (grammaticale e fisiconaturalistica), Herrera sovente offre la triplice equivalenza di ciascun vocabolo nuovo (greca, latina e castigliana, anzi spagnola, secondo l'idea herreriana della patria lingua messa in luce da Amado Alonso in Materia y forma en poesia, Ma- 
drid, I956, pp. 64-68); per es., nel son. XII di Garcilaso il poeta nota una sovrabbondanza di "sinalífas, que es el concurso de solas vocales; dicho propriamente en lengua latina elision, o colision, o concursion, i en nuestra herimicnton (p. I20); (questo significato di hcrimientlo, pur "desus.», sta nel dizionario dell'Acadcmia); altre volte sono proposti termini spagnoli clie non hanno avuto esito ("es el ipérbaton distracion, o trasgresion en la lengua Latina, $i$ en la nuestra, si le puecle caber este nombre, traspassamienton, p. IIo); ma trattasi sempre di atti inventivi non trascurabili.

Non meno copioso e risolutivo è l'apporto di liray $I_{4}$ uis de León al cultismo ispanico secondo il programma, coscientemente iniziato da Nebrija, della purificazione umanistica del barbaro e inquinato "romance" castigliano, nel quadro rinascimentale e europeo del classicismo volgare. Si pensi al virgilismo luisiano delle Bucoliche, cosi rigorosamente attento alla lettera testuale e a un preciso adeguamento formale inetrica italianista rispondente per dignità e prestigio alla metrica classica), rispetto al virgilismo epico di Juan de Mena o a quello enciniano delle stesse Bucoliche, violentemente adatta to alla realtà naturale e storica della corte isabelina e del campo castigliano, o a quello garcilasiano mediato e parzialmente corrotto dalla pastorale sannazariana. Primaria ̀̀ qui l'esperienza di traduttore dalla Bibbia ebraica, si che da fonti diverse per interna similazione si struttura la parola poetica delle odi originali. Alla confluenza di un complesso mondo ideologico e formale (biblicismo, italianismo, classicisno, neoplatonismo) si attenua alquanto l'antitesi linguistica tra $\mathrm{He}-$ rrera e Maestro León, di che daremo quelche esempio (tipi alpino, anibrósia, áspide, apolineo, roxo Apolo, avena, citara, inclemencia, inculto, empinar, fénix, furibundo, inclito, etéreo impirco, ecc.; termini esotici di piante ed aromi; ternini relativi all' ascensus neoplatonico; ecc.). La differenza sta, naturalmente, nel grada di intensità e saturazione. Rispetto alla tradizione romanico-ispanica si fa complementare il recupero del lessico arcaico secondo i diversi temperamenti poetici: Herrera è attento al tesoro "cancioneril" (tipi ensortijado, 
cusandecer, a/renta, porfía, cuita, listima, osadia, sañoso, raudo, fincar, conhortar, congoja, riel, ecc.), mentre F'ray I, uis tempera il suo arcaismo sul lessico devoto e didascalico di un Berceo ("linaje humanal, dardos herbolados, disanto, clanstra, basca. fornicio, naturales, fincia, abondo, ecc.n), che è ancora un capitolo inesplorato nello studio dell'arcaismo spagnolo.

Rispetto al lessico culto, avremumo desiderato un superamento più frequente e deciso delle mera nozione e preocupazione cronologica di "la documentación», e quindi una distinzione più netta tra cultismo fonetico e cultismo semanticoletterario, statisticamente valutato; ma qui un dizionario non si può improvvisare, necessitando alle spalle un tesoro di investigazioni monografiche ancora scarso e parziale. Si pensi alla metamorfosi semantica in Herrera e nei culterani di arcaismi come ardor delle Glosas de Silos, angélico o avena o corona o fantasia di Berceo, fucilar di Imperial, florido del Conde Lucanor, hebras di Fernández de Palencia, ecc. ecc. Proprio Corominas ci è di esempio; poniamo, per la voce ccríleo dà la "I. doc.: I427, E. de .Villena", ma aggiunge: "Fiué parte integrante y típica del vocabulario culterano, $y$ era ya vocablo favorecido de $F$. de Herreran; cosí per altre voci, cone ebrirneo, excelente, grado, industria, ecc. Per moltissimi vocaboli di questo tipo sarebbe stato opportuno far seguire alla prima documentazione da una fonte lessicografica quella dalla fonte di impiego letterario, di autore; ad es., diadema, posto che si trovi per la prima volta nel Vocabulario di APal (I490), si irradia specificamente nei testi poetici di Herrera (ediadema i cetro esclarecido", I, 9,60; "dorado diadema", I, 32,3) e di Góngora ("diadema de luciente Apolon); si consideri anche che Herrera attinge probabilmente al Vocabulario di C. de las Casas ( $\mathrm{I}_{570}$ ) e Góngora alla sctiola herreriana. Diamo una breve lista di altri vocaboli consimili, rimasti nella preistoria semantica meramente documentaria (trovansi tutti in Herrera e ne spiegheremo qualcuno tra parentesi):

accnto (canto), artifice, áspide, claustro, celsitud, concurso, 
conflicto, corage ('valentía', acc. etimol. nella c. III, 2,100), coyunctura, dardo, desamor, desconcierto, favor, furor, gloria, honor, acanto, adamantino, amaranto, electro, casia (II, 68,9), esmaltado, esmeralda, fanno, grifo, jacinto, acerbo, aneno, belicoso, caduco, confuso, culto, inculto ("incultos versos», II, 50, II), facundo, fogoso ("suspiros fogosos», el. I, 7,73; "fogosas flechas", c. I, 5,38), frágil, fugitivo (nimagen fugitiva", A, p. 304: "yo en el Faustino", v. 2, da "effugit imago" di Verg. II, 793), gallardo (el. II, 9,29), gcneroso, inclito, ingrato, inmenso, instante (in Herrera anche aggettivo inminente": "'1 mal instante", $I, 5^{8}, 5$ ), aspirar ("neologismo italianizante')) extirpar, incitar... Per voci come casida, diván, gacela, esperpento sarebbe stato bene annotare la loro fortuna di generi leterari nel 900 (L orca, Valle-Inclán).

Qualche osservazione sull'allestimento esteriore dell'opera ai fini di una facile consultazione.

Nell' elenco alfabetico delle Indicaciones bibliográficus occorre una certa difficoltà visiva, perché i cognomi seguonn ai nomi senza stacco tipografico.

S'è accennato alla legittimità del raggruppamento clei derivati, composti e affini intorno agli esiti etimologi fondamentali e significativi, ma la consultazione, non assistita da alcun accorginento tipografico, riesce piuttosto faticosa a chi sia inesperto di tali raggruppamenti per radici semantizzate, oltreché per dimenticanza o trascuratezza delle varianti ortografiche. Accade talvolta che una voce non stia nell'elenco: es,. afita, e confita trovansi in hito; argudarse in argiiir; baicquia in babieca; balanquin in baldaquin; hascas in casi; embellinar in beleño; fardido in ardido; escarvitar in escarbar; decoración, decorado e decorar ('acción de aprender de memoria') sono rinviati a decente come gli omonimi da decorum (forse ̀̀ un errore di stampa, giacché si trovano in coro III); aportunar starà in oportuno; controvadura in trovar; destojar in tojo; girgonça in jerga II; ambisa e ambiso in ier (non in avisar), ecc. Ma ripareranno gli indici finali.

Il dizionario è attento alle varianti morfologiche, na molte si desiderano; es., entrico, espeso, estido, escüi, ecc. 
Il rimettere alcuni prefissi (anti-, ccc.) ai composti porta a trascurare varianti di un certo interesse (ad es., nell'elenco alfabetico non esiste citra, quindi neppure citara, che Gómez Manrirue accentua parossitona: "Citara et vltramar" (Canc., 11. 4I7; v. Rosa Lida, p. 422) o a dimenticare neil'elenco voci organiche (ad es., auto che sta in acta).

Ier alcuni testi ci siano serviti delle seguenti edizioni: Calila e Dimna: Alemany Bolufer, Madrid, Igr5; Vida de S. D. di Derceo: Fitzgerald, Paris, I904; Milagros: Solalinde, $\mathrm{Cl}$. Cast.; Encina: Kohler, Bibl. Romanica; per Herrera abbiamo usato queste abbreviazioni: $B$ (ms. datato 1578 in Rimas inćditcs, a cura di J. M. Blecua, Madrid, I948), $H$ (eclizione in rita, I582); $P$ (edizione postuma, r6rg, entrambe nell'ediz. (iel Coster, Bibl. Romanica), A (Anotaciones, I580); per Iir. I,uis de León le Obras completas castcllanas, $2^{a}$ ed., a cura del P. Félix García, Madrid, I95r; con Rosa Lida ci riferiamo alla monografia su Juan de Mena, México I950; le citazinni da Góngora nel Vocabulario di Alemany; B. Potlier è il recensore del Coroninas in BH, I,VII, 4, I955; Gillet indica l'erliz. della Propalladia, Bryn Mawr P'ensylvania I943; $G$. de Díço è l'autore del Diccionario ctimológico español e hispinico, Madrid, I955; Cuervo: Diccionario, Instituto Caro y Cuerro, I (I953) e II (1954).

Un nostro studio stilistico, di carattere essenzialmente sincronico-estetico, su La lingua poetica di Fernaildo de Herrcra è comparso in due estratti dalle seguenti riviste: Preliminari e lessico da Studi urbinati, Anno XXIX, N. S. B. B. 2, Urhino 1955; Sintassi e metrica da Rivista cli letterature moderne, 1955 .

Seguiamo lo stesso metodo elencativo del Corominas; in mainsculo corsivo indichiamo i vocaboli aggiunti o retrodatati o esplicati nel significato o ncll'uso.

$\triangle B O C A D O$. Si noti il femm. A $B O C_{\text {r }} A D A$ ("...adrocata nostra...川), applicato alla Vergine da I'r. Luis de Leron ( $v$. qui 
la voce HUMANO) memore di Berceo, Loor., 220: "A ti façemos abogada".

ABISMO. I, a forma AUISSOS in Berceo, S. D. 24.

ABSTEMIO. "TEMULENTO [h. I580, F. de Herrera]"; sta già in Rodrigo de Cota; Diálogo, str. 34: "habla temulenta / turbada por senetud"; anche in Fr. I, uis, Los cantares, p. I707 (di incertissima attributione): "vino temulento".

ACORDAR I. Aggiungi $A C O R D A N Z A$, in Villasandino (Dezir de Alfonso Sánchez, Canc. Cast. s. XV, t. II, p. 379): "Pon ende, señores, con grant acordan fa...».

$A D O Q U E$. In Encina, Egl. eri requesta, p. 69, 'ornamenti nulliebri, perline': "dos mill adoques bermejos, / verdes, azules, pardillos».

AFECTO. "confección [confación, APal...; confacción, h. I490, Celestina...]”; CONFASI ON, Calila, p. I95.

AGRIJA. In Villasandino (Respuesta de Fr. de Baena, Canc. cast. s. $X V$, t. II, p. 369): «e que tenéis vna agrija / do la non queréys tener / por quanto podrié valer / toda vuestra escondrijan; V. GRADA.

AGUDO. "Agudeza [Berceo]"; AGUDENCIA in Mil. 225. AIRE. "Aéreo [ 1589 ]"; $A E R I O$ in Herrera (in $P$ come variante o lezione unica; es., c. II, I, 87; c. II, 5,75).

AI,A. Aggiungi DESALADO 'con le ali distese', in Berceo, Sacr. I77.

$A L B O$. "ALBA... 'tunica sacerdotal' [Gr. Conq. del Ultr.]"; in Berceo, Mil. 64.

ALHOLLA. In Calila, p. 364 (v. Glosario, p. 488).

ALIPERO. In Quirós (Canc. Cast. s. XV, t. II, p. 295): "que soys con vuestros leales / como leon y aliperos".

ALMA. Aggiungi il diminutivo ALMIELLA, in Berceo, Duelo 20.

ALMENDRA. ALMENDRILLA 'orecchino' (?) in Encina, Égl. en requesta, p. 69: "almendrillas y manillas».

ALMEXIA. 'Vestito, tunica', in Berceo, S. D. 669 e Duelo 20.

ALPES. "ALPESTRE [I582: Herrera]"; trovasi nell'ediz. postuma (el. II, 9, I45). "ALPINO [I589]»: anche questo 
agg. sta ini Herrera, $A$, p. 345, v. 25: "Alpinas cumbres", trad. dal latino di liracastoro, e in Fr. de León, Egl, X, 3I, p. I544: "sola y sin mí la nieve alpina helada".

AMAR. "DESAMOR [Nebrija]"; già in Berceo, -S. Lor. 25. Aggiungi CONTRAMOR, coniato su Anteros, in Herrera, el. III, 2, I23, e $A$, p. 322, v. 7 (e relativo commento a p. 320). $A M A R R A R$. Prima che in "Garcilaso" sta in G. Manrique (Canc. cast. s. $X V$, t. II, p. 40): "Avnque mis fustas (l.marron.

AMBROSIA. Si noti il latinismo tonico $A M B R O S I A$ in Herrera ("ila gracia de l'anbrosia parecia), $A$, p. 344, v. 28; "de celestial Ambrosia rociado", I, 40, 2; "ambrosia i netar espirò en su bocan, sest. I, 4, I8), in Fr. Luis, Pinl. I, I20, página I618: "la ambrosia que no pudo», e in Góngora. ANADIPLOSIS. In Herrera, $A$, pp. 95-96: uanadiplósis..., o redoblamiento, cuãdo la voz colocada en el fin del verso se torna a repetir en el principio del siguiente». Tale figura è detta propriamente epanástrofe (in DIPI,OMA trovasi "epanadiplosis»).

ANA'TOMÍA. "En la ac. de 'esqueleto' aparece anatomías ya en Cervantes"; in Fr. I uis de León, Del mundo y su vanidad (attribuita), v. I54: "Callo las otras muertes / de tantos reyes en tan pocos días, / cuyas fúnebres suertes / fueron anatomías, / que liquidar podrán las peñas frías».

ANCHO. "Cultismos procedentes de amplus...: AMPLO (SS. XVI.XVIII)n; già in Berceo: "Pero que ampla era la sancta vestidura", Mil. 72. "AMPLIFICACION", in Herrera (vedi qui IDIOMA).

ANDAR. Aggiungi $A N D A M I E N T O$, Calila, p. 403.

ÁNGEI. "ARCANGEL [h. I295: I.a Crón. Gral]"; in Berceo, Sacr. 8I. "ANGELICO [Berceo]"; si noti la diacronia cultista: Imperial - Herrera - Góngora - Lope.

ANOCEJA. In Encina, Égl. en requesta, p. 69: "Y frutas... / ... desas montañas: ... anocejas, refrisones...»; sempreché non si trovi in NUEZ.

ANTEO. In Encina, Égl. de Navidad, p. 27 'spavento': "Y de 
un ángel lo supimos. / Aunque gran temor ovimos / y nos puso gran anteo".

ANTIGUO. "ANTIGUEDAD [fin S. XIV, I,ipez de Ayala ]...»; in Berceo antiguadat, Loor. I43.

AÑO. "ANIVERSARIO [Partidas]..."; in Berceo alnnir'?sario, in senso etimologico (Sacr. 86) e 'tributo annuale' (S. M. 37I). Aggiungi ANNEL (Sacr. 149), e r. CORDERO, I, p. 903.

$A P O L I N E O$. Es., Fr. Luis de León, V, 37, "Gloria del apolineo sacro coro"; Verg., Egl. II, 6, II8: "que el apolinic" coro levantadon.

APORÍA. In Herrera, $A$, p. 78: "aporia o diapóresis".

APOSTROFE. "I. ${ }^{a}$ doc.: I632, La Dorotea"; giá in Herrera, $A$, p. 3 I5.

AQUILON. "I. a doc. S. XIV...»; B. Pottier retrodati it Fn. González, $4 \mathrm{r} 4$ a (aquilón) e I. ${ }^{\mathrm{a}}$ (rón. Gral. (aguilón), ma già in Berceo, S. Or. So.

ARCAICO. "ARCAISMO [Terr.]"; già in Herrera, $A, 1$ ). ror e I20: "arcaismòs o antigiiedad que llaman los latinns refuncion".

ARCEDIANO. "I. a doc.: ...arcediano, Partidus»; in Berceo, ARCIDIANO, Mil. 700.

ARDER. "ardura 'angustia, apuro' [Berceo, S. D. 453]"; aggiungi $A R D U R A D O$, Sacr. 272.

ARDIDO. "faldrido en Sta. $M$. Egipc.»; aggiungi FALDRIMIENTO, Calila, p. 92.

ARMA. "desarmar [Nebr.]"; in Berceo DESARMADO 'sturdito', S. D. 135 (nel testo $V ; E$ e $V^{2}$ leggono desarrado: $H$, desartado).

$A R N E R O$. In Frr. L. de León, Verg., Gíorg. I, 293, p. I55.4: "los debidos / arneros al Dios Baco" (lat. "mystica i'annus Iacchii). V. HARNERO in HARINA.

ARRE. "Canaria HURRIALLA (urri acai en Lope...)"; in Encina, Égl. en requesta, p. $7 \mathrm{I}$ : "Repastemos el ganado, / hurriallá!... Viénese la madrugada. / Hurriallí!); parinenti per le altre cinque strofe del Villancico; anche il semplice HURRIA, ib., p. 66: "Silva, ihurria! da gritillo". 
ARTICO. Prima che in "A Pal." sta in Villasandino (Canc. cast. s. $\mathrm{XV}$, t. II, p. 378): "Iil ártico polo".

ASAR. "ASADURA [II29 en el sentido de 'derecho...']"; in senso figurato in Berceo, Mil. 4I7: "Fizieron en don Cristo mui grandes travesuras: / taiava essa cuita a mí las assadurası.

ASINDETON. "I." doc.: APal.»; voci castigliane equivalenti in Herrera, $A$, p. 88: "asindeton... dissolucion, o desatamicnto... para dezir alguna cosa con ftuerça, vehemencia $i$ celeridad, con ira, impetu, amplificacion i grandeza».

$A S N O$. "Desasnar"; DESASNARSE 'divertirsi' in Encina, II Egl. en requesta, p. 74: "Y pues espacio nos vaga, / desasnémonos aqufn.

ÁSPID. In Herrera la forma $A S P I D E$ con -e paragogica conservata dal latino (già in Nebrija, fácile, dificile, fértile; felice in Antonio de Guevara; infelice in Góngora), utile metricamente: "Que, cual Aspide sorda 'l tierno acento"; anche in Fr. Luis, Job, XX, p. I0,39: "Cabeza de áspide mamará; p. I043: "que el áspide le beba sangre y vida». ASTRO. Aggiungi l'agg. ASTRUGO, Calila, p. 337 e Berceo, $S . D .423$ (in $H$ e in $V$ fo. anenturado); ancora in Berceo, Duelo I22, ASTRADO con lo stesso significato di "Astroso 'desgraciado...'".

ATOLA. ATOLEAR. In Calila, v. Glosario, p. 490. AUGUSTO. "I doc.: 1605, Picara Justina"; in Herrera, $B$, c. V, 26: "milagro angusto", e $P$, el. II, I,65: "que memoria augusta / pudo ser con mas onra celebrada?n; quindi in Góngora.

AÚN. 'Tra le varianti di aunque aggiungi $O N Q U E$, in Encina. Repclón, passim; v. Iamano, p. 556 che però non cita Encina.

AUTEN'IICO. Come agg. trovasi, prima che nel "Canc. de Baena", in Berceo, Sacr. 65: "Offrenda es auténtica...». AUTOR. In Berceo, oitre che "auctoridat", trovasi $A U C T O$ RIÇIA, S. M. 3II.

AVELLANADO. V. qui CIPARÁCEO.

AVENA. "Ia doc.: Berceo"; in questo autore ha senso pro- 
prio; manca la documentazione per il significato 'zampogna', frequente in Herrera: $B$, egl. III, 288; egl. IV, 239; $A$, p. 55 , v. I2 e p. 59, v. I; $P, I, 23,3$; senso proprio in $A, p .437$, v. 5 : "esteriles avcnas" (trad. di "steriles avenae» di Verg., Egl. V, 37); e in Fr. I. de I,eón da Verg., Égl. I, 2, X, p. 1505, Egl. VII, 38, p. I530 («docta avena), ecc.

AY. "I,a forma ay rie o $A Y M E$, usada en los clásicos [Malón de Chaide...] es de influencia italiana (ahimè)"; sta in Herrera, $B$, egl. III, 98: "Aymé mísero" c in $A$, pp. 22I-2, traduz. da Saffo: "i duermo sola yo, aime mesquina»; e vedi il commento sarcastico del Prete Jacopín in Obserraciones (Ediz. Bibliofilos Andaluces, Sev. I8;o, p. IS): A... Aquel me, me, una vez tras otra, es de poeta tan elegante como vos? Sin duda alguna aquel aymć mezquinu parece sacado de las razones que la nujer del Rei don Rodrigo dezía a el aya Clastras después de la inuerte de su maridon. Nella Seraphina di Torres Naharro, II, 2SI c 305, in italiano: "Oymê, Dio onnipotente... ;Oymê, Dio! e ti reng[r]acion.

AYUDAR. "Adyutorio... en el DHist.»; ADJUTORIO in Berceo, S. D. 220.

AJUMADO. In Berceo, Mil. 409: "Deso fablaban todos, mozos e ainmados"; v. ALJUBA, che è posteriore.

AYUNO. "AYUNAR [I24I, F. Juzgo]»; in Berceo, S. D. 62. BÁCULO. "I a variante baclo, en los Castigos de D. Sancho"; $B L A G O$ in Berceo, S. M. I4S; e i diminutivi BLAGUJELLO (S. D. I82) e BAGUILIELLO (ib. 709).

IBADALASA. In Encina, Égl. de Plác., p. I79: "Sus, ¿a qué quier jugar? / ¿Ballalasa, o rica pobre?" (v. Glosario: "juego antiguo. Cañete presume que viene clel italiano vala-lascia»).

BAFAR. BEFEZ. 'Di umile condizione', in Berceo, S. D. 55: "Qual non dirié nul onne, njn alto, njn befcz» (in $H$ e $V$; l'editore preferisce rafez di $E$. e $V^{2}$ ).

BALSAMO. "[1 doc.: fin S. XIII, I Crón. Gral.»; in Berceo bailssamo, Mil. 39. "embalsamar [I6+4, P. Manero]"; 
in Berceo BALSAMAR, Sacr. 27I: "Vinián al monumento a Christo balsamar'».

$B A N D A I I$. "en España es [ac.] casi exclusivamente nát1tica [lado... de una nave], mientras en América, y ya descle I'ernández de Oviedo [1577], se extiende hasta significar 'zona' y 'orilla, margen'». Questi ultimi significati sono costanti in Herrera (non in Góngora): "en la región suprema" (B) - "en esta vanda estrenı" ( $P, \mathrm{II}, 4,7$, cioè, la Spagna estremità dell'Europa); "en ambas vandas, / corre Istro" (A, p. 240, v. I); "el mar... / rodeando / sus vandas" (el. III, I,50); già nei Cancioneros: "las vandas de Poniente" (J. de Dueñas, La nao de Amor, str. 7).

BARATAR. "BARATO... adj., mod., 'de bajo precio' [Nebr.]"; in Berceo, Sign. 2r: "El día quarto décimo será fiera barata". "DES BARATAR ant. 'malbaratar' [fin S. XIII, I Crón. Gral.], mod. 'desconcertar, derrotar, descomponer' [I534]...); i due significati sono compresenti e fusi in Berceo, Mil. 878: "Despoiaron las sábanas... / libros e vestimentas... / fué nıal desvaratado el precioso lugarn, anzi è predominante l'impressione del sacrilego saccheggio, cui si subordina il motivo della "ganancia delgada" (877); in altri passi vale 'trucidato' ("dessent todos los otros fueron desbaratados", S. M. 292), 'cencioso, mal ridotto' ("Todo "lesbaratado, con pobre almexía", S. D. 728).

BARATRO. "infierno'... $\mathrm{I}^{\mathrm{a}}$ doc.: I6I2»; in Berceo, Mil. 85: "Por levarla al biiratro de deleit bien vazio".

BARBA. "Sobarba"; aggiungi SOBARBADA, in Quirós, op. cit., t. II, p. 303: "mas las grandes sobaruadas / que vos me dáys con frenon.

BARBECHO. "I a doc.: h. I300. Gr. Conq. de Ultramar"; lottier clocumenta la forma barueyton in Fueros de la Noienera, p. I22 (prima del I253); in Berceo, Loor. I84: "Segarán tales mieses qual fiçieron el barbecho".

BARBOTAR. "BARBULLAR [I" mitad S. XVI, C. de Castillejo; derivados en A. de Guevara]"; in Encina, Égl. de Plic., p. 167: "¿A qué vais allá? V'eamos / -A barbullar cierta trampa; / su preñez embarbullamos»; quindi si ag- 
giunga $E M B A R B U L L A R$; il senso dei due vocaboli è tolto dall'italiano imbroglio, imbrogliare, ingarbugliare (v. EiIBROLLAR).

BARRAGÁN I. Aggiungi BARRAGANADA 'ragazzata, impresa di giovinastri', in Encina, Repelón, p. I2I: "Al fin me hobon de caber / d'aquellas barraganadas / en las nalgas dos picadas, / que más ño pudon hacer».

BARRER. Aggiungi ABARRIDO 'saccheggiato'; in Berceo, $M$ il. 875: "Lo que fué en la ciella fué todo abarrido".

BARRUNTO. Aggiungi BARRUNTADOR 'spia'; in Berceo, Mil. 3II: "Methieron por la villa omnes barruntadores".

BASTO I. "BASTECER 'arreglar, disponer' ell Alex... y en fuero aragonés del S. XIII..., ac. general de la E. Media y predominante en el Siglo de Oro, hasta Cervantes»; questo verbo già in Berceo e implica un incontro di varie radici su una base omonimica (v. BASTIR, BASTO II, ecc.): 'provvedere, assistere' in S. D. 37 («entienden que el Padre sancto lo basteçio"), 'tramare, insultare' ("outo vn mal conseio aýna basteçido", ib. I64; "los que lo basteçicron ya eran repentidos", ib. I04).

BATIR. "Debatir [Berceo]"; aggiungi DEBATIRSE 'agitarsi', in Calila, p. $2 \mathrm{r} 8$.

$B A T R I C A J O$. In Encina 'colpo, fendente', Égl. de Antruejo, p. 6o: "y un batricajo le dió / tan cascante....); quindi in Lucas Fernández e, contaminato da bastón nella forma BASTRICAJO, in Torres Naharro, Ser., Intr. 63 (v. Gillet, III, nota a p. 234).

BEBER. Aggiungi ENBEBDARSE 'ubbriacarsi', Berceo, Mil. 463: "bebió mucho del bino, / esto fo sin mesura, / enbebdóse el locco...». Quindi manca anche BEBEDERO; ricordo di Fray $L$ uis de León: "No te engañe el dorado / vaso, ni de la puesta al bebedero / sabrosa niel cebadon, IX, I-3; manca in C. de las Casas.

BEFA. "I $I^{\mathrm{a}}$ doc.: princ. S. XVI: I,ucas Fernández»; in questo senso sta già in Berceo, S.M. 400: «Diçiendo grandes befas, dichos muy sobeianos». 
BÉLICO. "rebelar, Nebr."; Encina rustican!ente palatalizza in RE BELLAR: "Natco, si no rebcllas", Égl. de Navidad, p. 29, come rebellón 'ribelle' in Lamano, p. 599. Aggiungi anche BELIGERO, in Juan de Mena, Herrera, Góngora. BISODIA. E' dato Encina come " $\mathrm{I}^{a} d o c$ ", ma la definizione e l'etimologia ( il significato e l'uso del vocabolo (registrato per la prima volta); meglio recare un esempio dello stesso Encina, Repres. del Amor, v. 384: Mas mal de tales cordojos / no sé por qué causa sea; / qu'es una bisodia seria", cioc̀, 'un brutto affare, un malanno'.

BLANDO. "BEANDICIA", in Conedia de la Thebaj'da, página 40 (v. qui DEMULCIR). Aggiungi BLANDEZ in Calila, v. Glosario, p. 49r.

BIASFEMO. "BLASFEMIA", in Berceo, Duelo I92. Aggiungi $B L A S M A R, S . M$. IO2 (se non trovasi in IÁSTINA).

BOCA. Aggiungi $B O C A D A$, Berceo, S. D. 539: nnon podía comer vna nocada". "BOCADO [S. XIV]"; già in Berceo bocado (S. Or. 96; Sign. 33) e al plurale 'cibo in generale': "Saben con tal duelo amargos los boccados" (S. M. 35I). "boquirroto [ $2^{\circ}$ cuarto del S. XVI] ]; in Berceo, Mil. 285 , la forma $B O C A R O T O$ 'mala lingua'. Aggiungi $B O C U D O$ 'dalla bocca grande', Duelo 197.

BOCINA. "I doc.: Alex.n; BOCfN in Berceo, Duelo 49-50. BÓLIDO. "HIPÉRBOLE [I604, Jiménez Patón]", in Herrera, $A$, p. 133: "ipérbole, los Romanos le dieron por nombre superlación, o ecesso, o crecimienton.

BOI.SA. «Si fuese enteramente popular BŬRSA hubiere dado *bosan; infatti, BOSA si legge in Calila, p. 239: "E. vi que puso su parte dellos [los maravedís] en una bosa...».

BO'TA I. "BOTANA... [h. I500]", in J. Agraz (Canc. cast. del s. $X V, 11.447$, str.7).

BO'TIJA. Aggiungi BOTIJINA, in Encina, Égl. en requcsta, p. 69, tra le "frutas de mill maneras»; "cornezuelos, botijinas»; c v. Lamano, p. 295. 
BRAMAR. Aggiungi BRAMADOR, in Fìr. L. de L,cón, Job, IV, p. 870: "Al bramador león en un momento...".

BRAVO. In Encina, Egl. de Navidad, p. 3o, B\&RVEZ $\alpha$ per braveza.

BRAZO. Aggiungi la forma $A B A R \zeta A R$, Calila p. 42, e l'agg. verb. $D E S B R A Z A D O$ 'con le braccia distese', Berceo, Sacr. 63: "Demás quando estaba en la cruz desbraçado" (v. Cuervo, II, I003).

BROCADO. "Brocatel [I605], del cat. brocatell y éste del del it. broccatcllo, dimin. del anterior (brocatelo en 1535 viene directamente del italiano)"; BROCADELO in Quirós (Canc. cast. s. XV, t. II, p. 294).

BRUSCO II. " $I^{2}$ doc.: I5I4; I651... aparece una vez briksco 'tosco' en I ucas Fernández, a. I5 I4 ... podría ser entonces vocablo pastoril..."; in Encina, Égl. en requesta, p. 67: "grosero, lanudo, brusco!".

$B R U M A$. "Ua doc.: princ. S. XVII, Ruiz de Alarcón»; già in Herrera, $A$, p. 373 , v. 2: "elada bruma" (traduce "bruma -rigens» di Lucano, I, I7); $P$ III, 43, II (sostituisce sivierno" di $B$ ed $H$ ).

BUENO. "BONILLO 'lindo, mono', ya en la Gral. Est..."; in Berceo, Mil. 874. "BONITO [I5I7]...; BONICO, que aparece simultáneamente...n; in Encina, Repelón, p. I25: «Par Dios, bonicos estamos!»; p. I26: "que bonicos lo parorent.

BUHO. "BUHARRO 'ave de rapiña parecida al buho' [Nebr., aunque sin definición], otros ejs. desde $\mathrm{I}_{5} \mathrm{I}_{3}$; in Gómez Manrique (Canc. cast. s. XV, t. II, p. 40): "los que caçan con buharro".

BUSTO. "... parte superior del tórax'... $I^{\mathrm{a}}$ doc.: I605"; in Herrera, III, 35, 6: «el revelde busto / d'el impio que cayó con rayo justoi; manca in Góngora.

CABELLO. Aggiungi la forma DESCAPELLADO, Berceo, S. M. 220.

CABEZA. "DESCABEZAR [Nebr.]"; già in Berceo in senso proprio ("mandó... que lo descabezassen"), S. Lor., 45) e figut- 
rato ("Se tenien por muertos e por descabezados», S. $M$. 205).

CAJO. Agginungi CABAZÓN 'fine dell'opera', Berceo, S. Or. 202.

C.ICO-. "CACOFONIA [16r5]"; in Herrera, $A$, p. 233: "cacofonia o escrologia». Aggiungi ancora da Herrera, $A$. p. 89: "CACENFATON, O CACOFATON, o ESCROLOGIA, cuando se tuerce el sermón a entendimiento torpe, o por la juntura de las voces, que hagan mal sonido, o por la mesma significación".

CACHO I. Aggiungi RECACHAR 'dibattersi, lesinare', Encina, Égl. de Natividad, p. 24: "No son amos que se están / recachando en las meajas".

CAJA. " "I $I^{\mathrm{a}}$ doc.: caxa... Calila»; nell'ediz. Alemany, $C A[P] S A$, página 40.

$C A L M A$. Sulla "gran riqueza de las aceptaciones secundarias clesarrolladas en castellano" il senso traslato di 'angoscia, disperazione' prima che in "Lope, Tirso, Calderón", trovasi in Encina, Repelón, p. II9: "que me tomó una tal calma / que me pensé de transirn; v. Lamano. pp. 312-3.

CAMPANA. "Campanilla ['uvula': Nebr.]"; in Berceo, S. $M$. 485. CAMPANIELLA 'campanella': "Dues campaniellas pienclen sobre el so altar".

CAN. In Berceo la forma CHANES, Duelo 39.

CANAL. Aggiungi CANALADOR, in Fr. L. de León, Cantares (Respuesta), p. 202: «...aquella palabra (tsamatech) quiere decir también cabcllos, o lo que propiamente llamamos en castellanos en las mujeres copetes o canaladores" (la salmantina legge aladares).

CANCIÓN. Aggiungi CHANZON, Villasandino (Canc. cast. s. XV, t. II, pp. 364-5): "oyr cantar aues garrydos chanzones", "y con sones / de chanzones".

CANDELA. "CANDELERO [h. I300: Gr. Conq. de Ultr.]"; in Berceo, Sacr. 8.

CANDIDO. "ta doc.: Santillana»; per la fortuna dell'agg. presso i "cultos" (Herrera, Góngora...) notare meglio Juan de Mena ("cindida purpura", $72 e$ ). 
$C A N D O R$ "[Tirso]»; è vocabolo frequentissimo in Herrera.

CANORO. "U ${ }^{\mathrm{a}}$ doc.: Lope, Tirso"; in Herrera: "La canora armonia" (c. II, I, I6); "voz canora" (el. II, 9, 58); quindi in Góngora.

CANSAR. Aggiungi CANSADURA, Berceo, Loor. 82. "No se conoce variante alguna de la palabra cansar sin la $n \ldots$ ". ma vedi ENCASSADO 'rotto, stanco' in Berceo, S. $M$. 353: "Del andar e del planto fiera-ment encassados"; si metta questa forma in relazione con uel adjetivo canso 'cansado'... que recuerda el lat. QUASSUS 'quebrantado', o bien CASSUS 'carente, privado' (de ahí * ('sin fuerzas')", donde l'etimologia può vincere nel obstáculo insuperable de $\left.1 a^{*}-k t-\ldots\right)$.

CANTAR. "DISCANTAR [1599 G. de Alfarache]"; in Fray Luis de León, Pind. I, 28, p. I6I6: "Y con maestra mano/ discanta señalado...". "ENCANTO 'hechizo' [r6r5, Cervantes]»; sta in Herrera, el. III, 6, I68: "mas no es canto la voz, es fuerte 'ncanto'.

CANTO II. Aggiungi DESCANTO, in Berceo, Duelo rSo: "Non sabedes tanto descanto".

CAÑA. Aggiungi $C A \tilde{N} A V E R O$, in Encina, Égl. de Antruejo, página 6o: «heridos / a poder de cañaveros»; CANNAVERON in Lamano, p. $32 \mathrm{I}$.

CARBÓN. CARBONIENTO, aggiunto da B. Pottier (F $n$. González), sta prima in Berceo, Sign. I2, 'tenebroso'.

CARIÑO. "U doc.: $\mathrm{I}^{\mathrm{a}} \mathrm{I} 4$, Lucas Fernández»; già in Encina, Égl. de Carnal, p. 53: "Digo, hey, / tiene gran cariño al Rey"; Égl. en requesta, p. 65: "Qu'el cariño que te tengo".

$C A R M E N A R$. "El vocablo es muy popular hoy en día, sobre todo en el Oeste: salm. calmenar... 'injuriar'...»; in in questo senso in Encina, Repelón, p. II6: "que viene una milanera / tras mi por me carmenarn.

CARNE. Aggiungi CARONAL, Calila, p. 473. "Descarnar [Crón. Gral...]"; DESCARNADO 'macilento' in Berceo, $S$. D. 4 I5.

CARO. Aggiungi CARAMENT 'ad alto prezzo' in Berceo, S. M. 282. 
CARPIR. Aggiungi CARPELLIDO, in Villasandino ( $O p$. cit., p. 379): "dando garpellidos como gato en celo".

CARTA. Aggiungi CARTELARIO, sp. 'cartulario' in Berceo, S. D. I23; $C A R T E R A R I O$ nei testi $E$ e $V^{2}$; la prima forma sta anche nei $M$ il. 857 (adesque el cartclario fué cenisa tornado") ed è la "carta firme" della str. 740 , con la quale Teófilo aveva rinnegato Cristo e dato 1'anima al Diavolo, detta anche "cartiella" nella str. $8 \mathrm{r} 7$, cioè lo stesso foglio firmato.

CASAR. "Descasar [Nebr.]"; DESCASADO 'vedovo' in Berceo, S. Lor. 5 I.

CASI. "Enl cast. antiguo se decía hascas"; documentare con Berceo, S. D. $4 \dot{4} 3$ (cit. da M. Pidal, Origenes, p. 225); FASCAS, ib. I88.

CASTIGO. Aggiungi CASTIGUERIO, voce individuale di Fray L. de León, Job, V, p. 872: vy castiguerio del Abastado no aborrezcal.

CATACRESIS. Non documentato; sta in Herrera, $A$, p. 85 (traduce il lat. Abusio).

CAUTO. "CAUTELOSO 'engañoso, traidor', Lupe, La corona merecida, v. $22 \mathrm{I} 9$, y de ahí en dos traducciones del castellano al italiano, S. XVI, en Zaccaría.s. v.; la ac. moderna y latina 'precaución' no aparece hasta el Viaje de Turquía, I555"; il primo significato già in Cetina nel son. "Ved si el Amor, Señora, es cauteloson.

CEDER. "antecesor [I25I, Calila], de ANTECESSOR, id."; quest'ultima forma in Berceo, S. D. 54 e Mil. 68.

CEI,DA. "cellero, Berceo"; aggiungi dallo stesso autore $C E$ LLERER (S. Lor. 34) e ÇELLERIZO (S. M. 258).

CEI,O. C. de las Casas distingue celo, celoso ('gelosia') da ZELO ('zelo').

CENA. Aggiungi ÇENADA 'pasto', Berceo, Sacr. I5.

CENOBIO. Aggiungi CELOBICO, in Villasandino, op. cit., p. 377: "la vida çelóbica».

CENPELLIR. 'Lavorare con impegno', Berceo, Duelo 200 e $\mathrm{Mil.}$ 7I8; v. COMPELER e EMPELLIR.

$C E R C O$. Manca l'accezione di 'chioma', frequentissima in Herrera (non in Góngora). 
CICLAMOR. $\quad \mathbb{U}^{\mathrm{a}}$ doc.: F. de Herrera († I597), según cita de Alcalá Galiano...»; sta in $A$, p. 58, v. 7 ("el grande ciclimor») e in $P$, el. I, 8, x3 ("Ven, Ninfa, adonde el Ciclamor florece"); in $H$ si ha la forma CICLAMORO: "l'aura en los ciclamoros revestidos / de iedran (Egl. venat., v. I\&6; lezione guasta in $B$ : "la Aura, en los clamores revestidos»).

CIERTO. Aggiungi CERTERAMIENTRE, Berceo; S. $M$. 403.

CIMBRAR. "cimbrar puede ser apalear con vara delgada a alguna persona' [Atut.], y en este sentido figura ya el port. cimbrar, en el Canc. de Resendel; tale significato si documenta in Encina, Repelón, p. I2I: "E1 palo bien arrimado / cimbrado ña quella tiesta".

CIPERÁCEO. «derivado culto del lat. cypērum 'juncia'... $I^{\mathrm{a}}$ doc., Acad. I899"; aggiungi CIPERO, CIPRO, CO$P H E R$, in Fr. L. de León, Cantares, pp. I29 e 85: dopo aver tradotto in IV, I3 "juncia de olor y nardo" Fr. Luis nella "exposición» dà un equivalente (p. I29): "cipero y nardo" (lezione Merino; la salmantina legge "cipro"): e a proposito di "copher" in I; I3 spiega (p. 85) le varie versioni che se ne son date ("ipro" in San Gerolamo, "alcampfor", "alheña", "un cierto linaje de palma") col fatto che "no tenemos nombre para él" (v. anche Nonlbres, II); più avanti (p. I30) si dà la descrizione del ucipero": "...y en español se llama juncia de olor o avellanado, y en latín iuncus odoratus" (v. in JUNCO la definizione di Nebrija: "juncia: juncus odoratus, cyperus").

CÍTARA. "I doc.: I499, H. Núñez de Toledo... Más tarde aparece en Góngora y en Lopen; prima che in Góngora sta in Herrera: $B$, s. XX, 2 ("desta citara triste); s. XXX, 7; $A$, p. 4ro, v. 2 (da Ov., I, 559); $P$, el. I, 8,82 ("Que lira avrà, que citara llorosa...?»); el. II, I, 2; II, I5, 4; c. II, I, I2; e in Fray Luis de León: "la citara hería...», V, 34, "citara cantoran, III, I2, p. I433; Hor. L, I, 5I, p. I584. "Polimna / la citara me entregue / de I esbo»; Hor. III, 4, 6. página I533: "dulce citara», ecc.

"CITARIZAR, voz individual de H. Núñez de Toledo (r 499)", 
del quale si cita in nota: "citharizar es tañer la vihuela, que se dice citharan; tale verbo trovasi già in Juan de Mena (str. I20) insieme con CITARISTA (str. II6), documentato "I59I, Góngora".

CLARO. "Clarear [princ. S. XVII: Juan Hidalgo, Paravicino]"; CLARAR in Herrera, $P$, el. I, 9, 22: "Clarar la tierra i polo te conviene" (lezione in $B$ : "Dar claridad al orbe te conviene»); non sta in Góngora.

CLAUSURA. "INCLUSO [Covarr.]"; in Villasandino (Canc. cast. s. $X V$, t. II, p. 376: "la inclussa jaula".

CLEMENTE. Aggiungi INCLEMENTE (Herrera, es.: II, I08,7; c. III, I,84; c. III, 3, I09; III, 45,8) e INCLEMENCIA (Fr. Luis de León, XVIII, 28, p. I462; Góngora). de las Casas traduce l'it. sinclemente» con "sin clemencia». CLERO. "Clérico... variante semipolular, especialmente gallego-leonesa, crego (med. S. XVI: S. de Horozco; en el sayagués de Alonso de Solaya, Farsa...)"; CREGO in Encina (v. qui le citazioni nella voce FÍSICO).

COCER. "COCHO por 'cocido' (López de Ayala...)"; in Berceo, Sacr. I49: "Assado lo comiessen non cocho el annel"; Duclo 59: "Non serie tan cocha oi, nin tan asadan; il derivato "COCHURA", prima che in "APal", sta nello stesso Berceo, Duelo I64.

CODESO. "G. A. de Herrera..., y Fr. L. de León emplean el cultismo citison; dev'esserci un errore di stampa per citiso; es. Fr. L. de León, Verg., Égl. II, r, I46, p. I509: "vosotras ni del citiso floridon.

CODICIA. B. Pottier ha avvertito la mancanza delle forme medievali e cita COPDIÇIA (Sta. M. Egipc.); aggiungi Calila, p. I08, COBDIÇIA; Berceo: COPDIÇIA, CUBDIÇIA, COBDIÇIAR, COBDIÇIADERO, COBDIÇIO$S O$, ecc.

COGER. Aggiungere "DECOGER", in Calila, p. 483. Aggiungi DESCOGENÇIA 'scelta', in Berceo, S. Lor. 15. COLIRIO. "I555, Laguna"; CONLIRIO in Calila, p. 57, COLGAR. "DESCOLGAR [Nebr.]"; in Berceo, Mil. I5I, 'tirar giu (dalla forca)'. 
COLOSO. " "I doc.: 1587 , Ant. Agustín»; in Herrera, $P$, c. III, 3, 94: "Piramides... / ... / grandes colossos d'elevada cumbre»; quindi in Góngora.

COMA III. "[I6I7; Mariana]"; in Herrera, II, 69, 8 (gì in $B$ ): "utn ardiente cometa arrebatado"; el. II, 9, 35: "Las trenças... / que, cual cometa ardiente, resplandecen"; Herrera riceve l'antico vocabolo da Juan de Mena (cometas, (v. Rosa Lida, p. 260) e da Cetina (femm., "Las cometas que suelen señalarse / pronosticando cosa antes que sea", De la cola, vv. 55-56) e lo trasmette a Góngora. Proviene dai Cancioneros (v. ades., Villasandino, Canc. cast. s. XX, pp. $367,376,377,378)$,femminile.

CONCEBIR. Aggiungi APERÇEBIDO, Calila, p. II2, e APERÇE BIMIENTO, ib., p. 343.

CONOCER. Aggiungi CONOSÇEDORA, Calila, p. 427. "IGNORANTE [C. de las Casas; ignorante, I580, I. de Herrera, Coment. a Garcilaso]"; vocabolo ben noto nella scuola garcilasiana: Cetina lo riprende da un proverbio: "Amor lo hace y muestra bien ser obra / suya hacer que valga un ignorante / dichoso, más que un cuerdo desdichado" (son. El tiempo es tal, I2-14).

CONSEJO. Aggiungi DESCONSEJADO, in Berceo, $S$. Lor., 20.

CONSTRUIR. Aggiungi ESTROIR 'distruggere', Calila, p. 35I.

COPLA. "ACOPLAR [princ. S. XV, Canc. de Bacna: hablando de versos... ]"; in Berceo, S. M. 475: "Non los polemos todos [i nomi dei popoli...] en rimas acoplar».

CORAZÓN. "Cordojo."; aggiungi DESCORDOJAR, in Encina, Égl. en requesta, p. 7I: «descordoja tu cordojo", e DESCORDOJO, II Égl. en requesta, p. 78: "Pues aquí fué el descordojo / que pasamos hora un añon; Aggiungi DISCOR, in Villasandino (Canc. cast. s. XV, t. II p. 333): "que fazián por sus tenores / discores", (id., p. 363): "non rrompan ni despedaçen / los noveles sus discores".

CORRER. Aggiungi CURRICULO 'corso di studi', in COmedia de la Thebayda, p. 40 (v. qui DEMULCIR). "ESCO- 
RRIDO" in Berceo, oltre che "trascorso' ("noche escorrida", S. D. 367 e 588), significa anche 'aiutato': "Asmaban que en cabo serián bien escorridos", S. D. 408 (si tratta dei parenti dell'uenfermo", che sperano che il loro congiunto sia guarito dal "Padre benedicto"; in $H$, escurridos). "CORRENCIA [I605, Picara Justina]"; in Encina, Repelón, p. II7: "Que vos juro en mi concencia / que si mucho la studiara [la cencia], que más cara me costara / quizás que alguna correncian; Lamano, p. 354, segnala L. Fernández.

COR'TO. Nota 2: "[CORTON] En el Rim. de Palacio, 337 d, parece significar 'salteador, ladrón' ¿Uso figurado?n; tale significato è coufermato in una Respuesta di Pedro Morrera a Villasaudino (Canc. cast. s. $X V$, t. II p. 366): "Por cantones / con cortones / cantas e no te fallesçe...".

COSA. Aggiungi il significato etimologico, in Calila, p. 22: "... eran acordados... en las cosas delas enfermedades".

COSCORRÓN. "I doc.: ...I554', Lazarillo"; COCORRON in Encina, Repelón, p: I26: "Cocorrón que te daré!", con il suffisso rustico alla radice onomatopeica, come bobarón (da bobo), modorón (da modorra), baharón (da bafar), tutti aumentativi che leggonsi nella p. seguente del citato Aucto. COSER. "DESCOSIDO", in Berceo, S. M. 45I: "El su múy grant pueblo fu luego descosidon.

COSTUM BRE. Talora masch nell'uso culto, es. Fr. Luis de León (v. qui IGUAI).

COTA II. nexistió antiguamente una variante semi-popular CUTIANO..."; in Berceo come agg. (S. D. 397: "cutiana oración»; S. Lor. 32: "enfermedat cutiana»; S. D. 582: "dolores cutianos") e come avv. (S. D. 344: "Diéronli lo que dauan a los otros cutianon; Sacr. 95: La su sancta sangre cutiano la bebemos"). G. de Diego cita da $S$. D. 582 cutiano e rassegna la forma "COTIANO 'diario' ant. leon. Alex., I468\%. Corominas accenna al "derivado regresivo cutio (que G. de Diego acentúa arbitrariamente en la $i$, entendiéndolo erróneamente como descendiente de quottidio, Contr., párrafes $484-6) \%$. Alla stessa voce QUOTTTIDIO del Dic- 
cionario G. de Diego elenca le forme cutto e critio, cotio e cótio, rimandando alla Rev. Port. de Filol., 5, I, 2.45-6. V. anche il Registro di Romera-Navarro alle voci CO'IIO, CUTÍO, CUTÍO (DE).

COTURNO、 "I ${ }^{\mathrm{a}}$ doc.: APal...; princ. S. XVII (Góngora; Pantaleón, Armaya, en $A u t$.)»; è sempre Herrera che per primo a tali vocaboli dà cittadinanza di poesia: $B$, c. V. 73 , e $A$, p. 4r4, v. 4: "tus versos, / dinos solos del coturno / de Sofocles" (traduce Verg., Egl. VIII, Io: "sola Sophocleo tua carmina digna cothurno?").

COZ. "COCEAR [Nebr...]"; in Berceo, S. D. Ioz e S. Or. 30. CRECER. "DESCRECER: Fr. Luis de Granada, Cervantesi); in Berceo, Loor. I83.

CRESPO. "CRISPAR [Acad. I884, no I843]"; è preferenza herreriana dell'ultima redazione, usato come sost.: in $P, \mathrm{I}, 74,9$ sostituisce "centellear" di $H$ ("el ardiente crispar de dulces ojos»); c. I, 5, 40 (《i d'el fulgor hermoso al crispar tierno"). Aggiungi CRISPANTE, P, I, 20, 2 ("crispante cabello"). "crespar [S. XVI-XVII, Avt.]"; sempre in Herrera, 1'agg. CRESPADO, $P$, el. II, I2, 74 ("crespadas ondas»). Vieppiu si nota che la postuma per secoli è rimata inutilizzata.

CRIMEN. "Crimen... I $I^{\text {a }}$ doc.: Berceo... CRIMINAL [APal... Nebr.]"; nello stesso Berceo: "pecado criminal", S. D. I37. "CRIMINOSO [A Pal...! Nebr.]"; sta prima in Diego del Castillo, Visión, str. 43. "creminoso".

CRIN. "CRINADO. CRINAR.»; documentare con Juan de Mena ed Herrera: c. II, I, I3 ("crinado Apolo"), cl. I, 9, I ("Rubio Febo crinado»), el. II, I, Ior con l'accezione 'ornare la chioma, incoronare' ("crinò mi frente 'l arbol de vitoria)).

CRUZ. Aggiungi DESCRUCIJAR 'separare le braccia incrociate', in Berceo, Sacr. 208.

CUARTAGO. " $U^{\mathrm{a}}$ doc.: Lope de Rueda...»; Fr. Luis de León traduce "mannos» di Hor. III, 27, 9, p. I607 (lezione Merino): "al cuártago temiendo». 
CUBO. Aggiungi GUBEL 'recipiente', in Juan Agraz (Canc. Cast. del s. $X V$, n. 446 , str. 5).

CUELLO. "CUELLIERGUIDO [1605, Picara Justina]"; in İr. L. de León, Verg. Georg. II, 265, p. I577: "De aquí el guerrero potro cuellierguidon (lat. "arduus»). Aggiungi.

CUERPO. "CORPOREO [h. I640; Nieremberg]"; in Herrera, $H$ e $P$, el. III, 8, I6: "corporeo velo"; aggiungi INCOR$P O R E O$, in $A$, p. I64: "es nuestra anima un espiritu incorporeon. "Corpulento [APal. $95 \mathrm{~d}$; CORPORIENTO, 50 d]"; quest'ultima forma in Calila, p. 380. "Corpanchón... corpachón... corpanço (1464), Mingo Revulgo; ...'cuerpo de ave despojado de las pechugas y piernas' Rob. de Nola...)... Corpazo..."; CORPANCHO in Encina, Égl. de Plác., p. I8o: "...esa cesta de paja... qu'el corpancho me deleita / y me suele gasajar"; CORPORAR 'ingrossare', Lamano, p. 353. CUAJO. "Descuajar... [Ribadeneira † I6II]"; in Berceo $\mathrm{i}$ p.p. $D E S C U A I A D O$ (S. D. 45I, in tutti i testi) e $D E S$ CUIADO (Mil. 9I).

CUCO. "Cucar 'mofar' [h. I500: J. del Encina, cita en Lamano]...»; Encina ha anche CUCUO, in Égl. de Plác., p. I68: "Cuitado del desposado, / que es ante cucuo y cornudo".

CUELLO. "COLLARADA 'parte de una camisa' [J. Ruiz...]" , in Calila, p. 217, agg. ("paloma collarada") e p. 2I8, sost. ("la collaradan). DESCOLLADO, e vedine il significato e le citazioni (tra cui Fr. L. de León, Cantares, p. r26) in Cuervo, II, I003.

CUERNO. "CORNAL 'coyunda...' [Acad. ya I843, como voz provincial, con variante cormil]"); in Berceo 'lato dell'altare', Sacr. 50: "A los moros significa el siniestro cornaln. CULO. Aggiungi DESCULAR, in J. Agraz (Canc. cast. s. $X V$, n. 447 , str. 8): "tinajuelas desculadas, / que se llaman coladeras".

CULPA. Aggiungi la forma COLPA, in Calila, p. 346.

CULTO. Nel significato letterario .[I607, Oudin]... Góngora desde I6I3"; sta naturalmente in Herrera, come lezione unica o come variante nell'ultima redazione di $P$ : el. I, 6, 87 ("culto Lasso"; elegia anteriore al 5557 , ma probabilmente fu 
rielaborata); I, 64, 4 ( $B$ : "torna, Amalteo, a resonar th lira» - $P$ : "te guia, osa herir tu culta lira»); c. II, 2, 48 (H: 1noble i docta lira» - P: “culta noble lira»); el. II, 7, I34 (H: "Lasso" - P: "culto Lasso"). Quindi anche INCULTO: c. III, 3, 5I: "inculta Musa mia»; Fr. Luis de León, II, 3I, p. I 432. "En pueblo inculto y duro..." "CULTURA [I583-5, Fr. L. de León]"; ha significato di 'culto religioso' (es., Casada, XI, p. 305: "cultura de idolos»), oltre all'usuale 'coltivazione' (es., Nombres, I, 2, p. 520: "cultura del campo"). CUMBRE. "CUMBRAL ast. 'la viga que se pone cu la cumbre de un techo'»; in Berceo, Mil. 323: "ardieron... las vigas... los cumbrales" (Solalinde in nota cita un passo del Fuero de Soria II: "nin por cumbral, nin por forquiella...". CHAPA. "CHAPADO, aplicado en son elogioso a las personas (frecuente en las églogas del S. XVI)...); si noti l'estensione semantica dell'agg.; per es., in Encina, oltre che 'bello elegante, ecc.', significa 'grave, eccesivo': "I'iene a la mi fe, señor / mal de amores / de muy chapados dolores", Repr. del Amor, v. 306. Aggiungi l'avv. CHAPADAMENTE, nello stesso Encina, Repelón, p. I22: «Sí, clapadamente huyen / si tras ellos va algun canto». "chapar... su uso es mucho menos frecuente que el del sust. (faltan ejs. en Aut)"; CHAPARSE 'chiudersi, rinserrarsi' nel cit. Repelón, p. I23: "que se chapen llugo en casa / primero que ñada habren*.

CHAZAR. "CHAZA 'suerte de juego de pelota...', que ya aparece en Guevara (I539...); in Encina, Repelón, p. Irf col senso traslato di 'scherno, canzonatura': "entró 111 estudiante... a refacer la chaza».

CHICO. Aggiungi CHIQUINEZ 'fanciullezza', in Berceo, Mil I75.

CHIRIVIA. «I doc.: cherevia, Berceo, S. D., 70 d»; il testo $V$ legge chirivias, e nella str. 378 dello stesso poema: "Nas rancar non podieron puerro njn chiriula» (in $H$, cheriutia).

CHOLLA. " $U^{\mathrm{a}}$ doc.: 1509, Juan del Encina, Auto del Repelón (cita de Cuervo...)"; sta prima nella Repres. del Amor 
(I497), v. I66 (v. qui la voce HERIR); per altre citazioni v. Gillet, III, nota a p. 353 e Registro di Romera-Navarro.

DALMÁTICA. . In Berceo, S. D. 232, ALMATICA.

DAÑO. Aggiungi CONDENAGE, in Villasandino Canc. cast. s. $X V$, t. II, p. 365) in un gioco di rima in - age.

DAR. "APODOSIS [Aut.]"; in Herrera, $A$.

$D E B E R$. Aggiungi DIUDO 'innamorato', Calila, p. I78: "e avía un vezino pintor e era diudo della".

DECIR. Aggiungi $M A L D I C I O N$ e quindi $D I C I O N$ con lo stesso semantema (opposto a Bendición) di 'macchia, peccato', come in Berceo, Mil. I8I (1la Gloriosa plena de bendición, / es plena de gracia, e quita de diciónn) e 228 ("La Virgo Gloriosa, madre sin diciónn).

DELANTE. "Enanzar 'avanzar', nav. y arag. ant..."; in Berceo, Sacr. I93, in senso traslato di 'profittare': "Amigos, estas cruces que en cabo contamos, / cinco fueron por cuenta, ca bien nos acordamos, / en la hostia por ella nada enançamos, / mas las plagas que fueron en Christo remembramos"; il vocabolo ha $-\varepsilon-$, come in "cat. ant., oc. ant».

DELEBLE. "I ${ }^{2}$ doc.: falta todavía Acad. I899"; "Deleto, ant.»; in Berceo DELEIDO: "que sanó tan aýna cosa tan deleida", detto di una paralitica (S. D. 590).

DELIBERAR. "I ${ }^{\mathfrak{n}}$ doc.: delibrar... Sánchez de Vercial, † I426»; in Calila, pp. ro4 e 260, si definisce l'uomo DELIBRE, cioè 'attivo, intelligente, coraggioso'.

DEMONIO. nendemoniar [S. XVII: Aut.]"; ENDIMOÑAR in Encina, Egl. de Plác., p. I79: «los de villa y palaciegos! / El amor los endimoña.

DEMULCIR. " "I ${ }^{a}$ doc.: Acad. ya I8I7"; nella Comeaia llamada Thebayda (152I); voce segnalata da Menéndez Pelayo, Origenes, IV, p. 40.

DESMANI. ulesmanar... 'dispersar, desbandar' en APal... desmanarse 'descarriarse, separarse del rebaño'... en $\mathrm{Ne}$ brijan; DESM ANAR 'allontanare, evitare' in Calila, p. 476: "el religioso... me compró [parla il "gato"] por dcsmanar el dapĩo que le fazías...». 
DESNUDAR. Aggiungi la forma rustica DUSNAR, in Encina, II Egl. en requesta, p. 84, e Egl. de Cristino, p. I43. DESPEDIR. Aggiungi DESPIDIMIENTO 'partenza', in Berceo, S. M. II6.

DESTELLAR. "Destilar [...Celestina...]"; si noti la variante herreriana DISTILAR in $A$ e in $H$ (destilar in $P$ ); sta già in $\mathrm{C}$. de las Casas e passa a Góngora.

DÍA. "Diöso [S. XIII, Bocados de Oro; I605, Picara Justina]"; in Calila, p. 304 (sul significato del vocabolo v. Glosario, p. 494).

DIAPORESIS. In Herrera, $A$ (v. qui APORÍA).

DINERO. "DIN [Espronceda, $+\mathrm{I} 842 \ldots]$ ]; in J. Agraz, cit., n. 450, str. 2: "pobre / manzollado sin dines".

DIOS. "divinal [Nebr.; APal...]"; DEVINAL in Berceo, S. D. 486.

DIPLOMA. V. qui ANADIPLOSIS.

DISCO. " " $I^{3}$ doc.: Covarr., con referencia exclusiramente a la Antigüedad"; in Herrera, $A$, p. 275 , v. I8: "Llevando la vitoria / con disco, i dardo, que traspasse '1 termino?" (trad. da Hor. I, 8, II-I2: "...saepe disco...»).

DISPENDIO. "Expensas [I494, Regimiento de Principes]"; EXPENSA in Berceo: "Comno facié grant gasto, espensa sin mesura", Mil. 630. Aggiungi ESPENDIDO 'crocefisso', Duelo 220.

DOCTOR. Aggiungi la forma dialettale DUTOR, in Eincina, Repelón, p. I20: "ni habrar como dutor».

$D O M A G E$, in Berceo, S. M. 44I: "Fiçieron tal donnage cu los mas delanteros».

DONAR. "DONAIRE [h. I250, Setenario...]"; in Berceo, Loor. 224. DONOSO in S. D. 443 non è "'generoso", ma 'pieno di grazia': "Dixo por elli missa el donoso sennor"; non vi è "significado vago" in Mil. 25, ma senso effettivo di 'piacevole": "Los árbores que façen sombra dulz e donosa»; 'miracoloso' in Sacr. I29: "Una hostia fué sola, essa fué tan donosa, / que nos quitó a todos de prisón peligros(l) ( $\mathrm{si}$ noti in rima l'opposizione donosa-peligrosa).

DORMIR. "Adormidado... dormitar [Berceo]»; anche 
ADORMITAR (S. M. I0), ADORMIRSE (S. D. 652) e $A D O R M I D O$ (Mil. 255).

DUEÑO. Aggiungi DOÑATA, in Encina, II Égl. en rcquesta, p. 8I: "Qué te parece, Menguilla, / de cuál está Pascualeja? / Dóme a Dios que ya semeja / doñata de las de villa. / Miefé, ya se nos engrilla!n. Aggiungi CONDOÑO, in Villasandino (Canc. cast. s. XV, t. II, p. 369): "Marchito padre condoño / letuario contrafecho...».

DULCE. La forma "DUÇE" si documenta in Calila, p. 44.

DURAR. Aggiungi ATURADAMENTE 'amaramente', Berceo, S. D. 392.

DURO. Aggiungi DURIÇIA, in Berceo, Sacr. 294.

ECHAR. "desechar /Berceo...]"; DESENCHAR, Calila, página 236.

EMBARAZAR. "baraza... en C. de las Casas»; leggasi "ba$r a z$ - imbroglio, impedimẽton; manca nella Prima Parte. EMPINAR. "I $I^{\mathrm{a}}$ doc.: Nebr... Es frecuente en autores desde Argote de Molina (1582) y principios S. XVIIn; in tale frequenza vengon prima Fr. L. de León (Job XXII, p. I066: "La cerviz erguida / que tanto se empinaba, vino a tierra", XXXIX, p. I260: " $¿$ Si bate y tiende / las alas renovadas y se empina?"; 'esaltare' in Georg, II, I78, p. I575: "Mas si te empino...川) ed Herrera: $H$ e $P$ (el. I, 8, 32 e c. III, 2, 70: "empinados arboles"; III, 60, 2: nempinada sierra"); solo in $P$ (c. III, 6, I48: "empinados montes»); preferenza in $P$, I, 76,9 ( $B$ e $H:$ :A un lado levantan su grandeza / los riscos" - $P$ : "A un lado empina ierto immeusa cumbre...".

EMPÍREO. "V. piro-»; la forma IMPIREO è herreriana prima che gongorina; anche in Fr. Luis, $A$ la vida religiosa (attribuita), v. 45, p. I495: "la gloria eterna del impireo cielo".

ENCENDER. Aggiungi da Berceo le forme ENCENSARIO (Sacr. 35) e ENCENSERO (Sacr. II9).

ENDIADIS. " " doc.: Acad. I884, no I843"; in Herrera, $A$, p. 375: "endiadis, figura... que el nombre está en vez del ayuntadon.

ENFERMO. "FERMERIA" in Berceo, Mil. 245 col senso 
traslato di 'luogo di dannati, inferno': "Vaia yacer con Iudas en essa fermerial.

ENGATUSAR. "GARATUSA 'caricia, halago' [Espinel, I6r8; Tirso...]; in Encina, Repelón, p. II8.

ENHIESTO. Aggiungi INFESTO, in Herrera, A, p. 546, v. 4: "Cuanto el vigor infesto de la mente" (traduce "Quacumque mentis... infestus vigorn, Sen., Oct., v. 740).

ENTRE. "INTIMO [h. I580, Fr. L. de Granada]"; in Herrera, $B$ e $P, I, 47$, I4 (cf. $P$, II, 44, I4): «i el crudo golpe desta acerba llaga, / al'intimo llegò de l'alma mia». "Intestino [I59I], Percivale, en el sentido de 'tripas'; I6II, Covarr.; estentinos, Alex..."; ENTESTINO, in Calila, p. I95: "fueron los sus entestinos despedaçados e [senza corrispondenza nel testo arabo] murión.

EPANALEPSIS. Si documenti con Herrera, A, p. IOr: "epanalésis, que llaman los Latinos refuncion, la cual es una apartada buelta $\mathrm{i}$ repeticion de verbo con algunas vozes interpuestas; porque buelve a tomar lo que avia dexado..."; per la distinzione dalla uepanáfora» v. p. 97.

EPANODOS. In Herrera, $A$, pp. I74-5: aregresion o reversion, dicha de los Griegos epanodos i de nosostros rebueltan. EPITROPE. Potrebbe stare in TROPO; in Herrera, $A$, p. 86: nepitrope... permisionn.

ERRAR. "ERRANTE [Quevedo]"; in Herrera, P: nerrante furor» (I, 58, -IO), "errante peregrino» (II, 4I, 6), "aire errante» (el. II, 6, I34; in $B$ : "aire errando", donde è lampante il transito al participio culto); quindi in Góngora. "ERROR [Berceo; 'ofensa, agravio' en el Rim. de Palacio... ]"; in Berceo significa 'vizio, peccato'. La Rosa Lida, p. 363, rileva in Herrera, el. II, 5, 46-48, il significato latino di 'enredo, confusión': «el oro en rico cerco recogido, / con bello error entorno, descompueston; si tratía delle chiome di Luz graziosamente scompigliate, immagine che passa a Góngora: «...a la Nimpha mía / ...ondeábale el viento que corría / el oro fino con error galano».

ESCABROSO. "I $I^{\mathrm{a}}$ doc.: Ambr. de Morăles (Aut.)»; in 
Fray L. de León, Salm. CXIII, 36, p. r66g: nescabroso / estéril riscon.

ESCONDER. Un aggettivo verbale analogico a un passato remoto forte dev'essere "ESCOSA" in Calila, p. 48: "Et quando ha andado eneste mundo, torna viejo et a escosa e desabrida vidan, quindi un omonimo dell'agg. femm. es$\cos a$ da EXCURSA (V. ad v.).

ESCROLOGIA. Manca. V. CACO.

ESCUDILIA. Aggiungi ESCUDILLO, Calila, p. 369: "su escudillo de oro enla mano conel arroz..." (v. ESCUDO).

ESCURRIR. "ESCORRECHO 'sano, no lisiado, en los Castigos de D. Sanchon; in Calila, p. 349: "Ca el omne maguer sea esforçado e escorrecho....).

ESMLALTE. Aggiungi DESESMALTAR, in Quirós, op. cit., t. II, p. 30r: "el dolor que desesmalta/mi vida con la tristura".

ESPIGA. Aggiungi ESPIGADA, in Berceo, S. D. $45^{2}$ (espigas in $H$ ).

ESPINA. Aggiungi ESPINAZA 'spinacio', in Berceo, S. Lor. 87: "Mas sabrosas me saben que unas espinazas».

ESPIRAR. "espirtu en versos de Hurtado de Velarde y Fdo. de Herreran; in Herrera espiritu compare rare volte e solo in $P$, alternando con espiritu per ragioni metriche; per es., "el espiritu os halla, i tanto veo" ( $A, \mathrm{p}$. II6, v. I2)"el espirtu vos halla, i tanto veo" ( $P$, est. $I, 2,3)$. Aggiungi ESPIR ACULO, in Herrera, $A$, p. r64: "es nuestra anima... centella y espiraculo de la mente divinan; prima fonte Virgilio.

ISTAMPAR. "ESTAMPIDO 'ruido fuerte y seco' [1607, Oudin; I6II, Covarr.; I6I3, Cervantes]»; in Herrera: «arroja contra el cielo enfurecido" $(B)$ - "arroja con orrisono estampido ( $P$, el. III, I, 66, databile $\mathrm{I58 \textrm {I }}$ ).

ISSTAR. "DISTANTE [h. I60o, Mariana...]"; in Herrera, $P, \mathrm{II}, \mathrm{I} 4$, I57: ni ardor puro / distante, pero cerca se m'asconde»; quindi in Góngora. Aggiungi la forma italianizzante ESTANZA, in Fr. L. de León, Nombres, III, Intr. p. 663: "en la huerta había estanza fresca y apacible"; 
Verg. Egl. II, 9, 70,p. I540: "Aquí haze la vid estanza umbrosan; Herrera conserva l'antica grafia ESTANÇA per significare la forma metrica italiana.

ESTIMAR. Aggiungi ASMADUERO 'concepibile, immaginabile', in Berceo, S. M. 306.

ESTRELIA. Aggiungi COSTELADO, in Villasandino (op. cit., p. 372): "Porque fuy mal costelado, / ssabe Dios que me manzillo.

ESTRIBOTE. Pierre Le Gentil in La Poésie lyrique espagnole et portugaise a la fin du Moyen Age, $2^{\mathbf{2}}$ Partie, Rennes I953, pp. 22 - 232, espone una tesi diversa da quella del Corominas $\mathrm{e}$, in genere dalla teoria ispano-andalusa di Menéndez Pidal (in particolare, il riferimento al Diccionario nelle pp. 23I-2).

ETE,R. "Ettreo [Lope]»; in Fr. Luis de León, Job, II, p. 840: Ábrese ya otra vez la etérea entradan; in Herrera ETERIO, in $P$ come variante o lezione unica (es., I, 6, Io; II, I7, 6; c. II, I, 27; c. II, 2, 58; el. III, I, I26); quindi in Góngora.

ETEROSIS. Figura di enallage, dal greco; in Herrera, $A$, p. 87: «eterósis cuando varian los casos i generos, los numeros i personas, los modos i tiempos".

EXAGERAR. "Exageración ['encarecimiento'], Quijote...»; ESAGERACION, figura rettorica, in Herrera (v. qui IDIOMA).

EXCELENTE. "Excelencia [Celestina...]"; in Juan de Mena, EÇELENCIA (26 a). "Excelso [princ. S. XVII: Lope, Pant. de Ribera]"; in Herrera frequente ECELSO.

EXHAUSTO. "I ${ }^{\mathrm{a}}$ doc.: I6I4, Aldrete»; trovasi "INESAUS$T O$ fuego" in Herrera, $P$, III, 4I, II (lezione affatto distinta in $H$ ).

FALDA. Manca l'accezione 'parte inferiore di un monte', registrabile in Fr. Luis de León, Cantares, VI, I, p. I54 ("...en los arrabales y aldeas, que estaban a la halda...") e in Herrera, I, Ir7, I-2 ("La falda, i el tendido, ierto lado/ d'el abrasado Etna"), quindi in Góngora ("faldas Pyre- 
neasii), che l'usa anche per 'riva' ("rel Eritheo / besa a Arabia las faldas olorosas").

FALIIR. "falible [Nieremberg, I658]"; INFALIBLE in Herrera, el. III, 2, r29 (ai este es decreto d'infalible assientoin), quindi in Góngora.

FAMIA. Aggiungi DISFAMOSO, in Villasandino (Dezir de Alfonso Sánchez, Canc. cast. s. XV, t. II, p. 379): "disfamoso conponedor.

FANTASIA. " "Ioc.: Berceo, Mil. 433 b»; in altro passo (S. D. 70) al plurale col significato di 'circostanze': "Suffriá fiero lazerio las noches e las días, / tales como oyestes en otras fantasias». Correggi "Mil. 443 b.».

FARSA. "I ${ }^{2}$ doc.: I520, H. López de Yanguas, Farsa sacramcnlal en coplasn; dello stesso autore la Farsa del mimdo; ma anteriori sono le sei Farsas y églogas al modo y estilo pastoril y castellano, Salamanca I5I4, di Lucas Fernández; si ricordino le Farsas (del I505) di Gil Vicente.

FATIGAR. "FATIGOSO [I605, López Pinciano]"; in Herrera, $A$, p. 344, v. 30: ai linage afanado i fatigoson.

FAUSTO. "pompa, lujo...' .... Ia doc.: FASTO, Sánchez de las Brozas, $t$ I600»; anche in Herrera, el. III, 5, ro: "De l'ambicion el fasto i la grandezan; Góngora usa FAUSTO, documentato " 596 (Fonseca)".

FAVOR. "Fausto, adj. [h. I600, Argensola]"; aggiungi INFAUSTO, sempre in Herrera, $B$, el. III, 43: "Infausta fué a mi vista su bellezan.

FELIZ. Aggiungi la forma INFELICE (v. qui la voce ÁSPID).

FENIX. " $u^{\mathrm{a}}$ doc.: $1570, \mathrm{C}$. de las Casas ("fenice: aue fénix"; falta en la parte cast. - it.); I582-5, Fr. Luis de Granada... Agrego un ejemplo de Lope: "...la fénis muerta....; in Fray Luis de León, Quien viere, v. I8, p. I493 (attribuita): upues la fénix que solo tuvo el mundon; in Herrera, di genere maschile, $B$, est. I, 25 ("Purpúreo fénix que la Arabia cría") e $A$, p. 52, vv. 6-7 ("i cuanto el encubierto / Fenis quema». Ambigenere in Góngora.

FETILA. 'Freccia dolorosa' in Berceo, Duelo I3: "Pero la 
mi fetila non la he oblidada / ca en el corazón la tengo bien fincadal; aggiungi anche $F A T I L A D O$ 'angosciato', $S . M$. 455 e FETILLADO, S. Or. r86, FAZILADO 'trapassato dal dolore', S. M. 355 ("corazones... fazilados») e FEZILADO, S. M. 205 .

FIAR. "FIADURA [J. Ruiz...]"; in Berceo, Mil. 680: "Nunqua omne non fizo tan loca fiadura / que priso por fianza una imagen dura».

FIN. "FINIQUITO [1554, N. Recopil., ell Aut.]"; in Encina, Repres. a la Pasión, p. 42: "Oh gran don / de carta de finiquito / para nuestra redención!!; v. Lamano, p. 462.

FINGIR. «U doc.: APal... Enfingir [enfiñir, $I^{\mathrm{a}}$ Crón. Gral...]"; alla coniug. di $E N F I N I R$ deve appartenere il perf. enfinió (estraneo al testo arabo) in Calila, p. I83: "Los omnes que son tales [falsi] son cinco: ...et el quarto es el huésped que se enfinió et cúyda que es señor dela casa...". "EFIGIE [I615, Quijote...]"; in Herrera, B, el. IV, 56: "ry llevo vuestra efigie en mis entrañas»; in $A$, p. 347, v. I: "bella efigie». FINO. "AFINAR [I288...]"; in Berceo, S. M. 420; "El rey don Remiro... Afinó un buen consejo».

FIRME. "Firmedumbre [...Calila...]"; la forma è FERME$M E D U N B R E$, p. 497 , testo $B$; aggiungi FERMEDAT, ib., p. 244: Rilevare il significato di 'solito, abituato', in Berceo, S. M. 43: "Rézando so salterio que abié $A F I R-$ $M A D O$ \#; aggiungi $A F F I R M E S$, Duelo rg9: "Affirmes trabaiary. FIRMEDUMBRE sta prima in Berceo, Loor. 80. FÍ́SICO. "La ac. comun en la Edad Media es 'médico...' (Calila... Nebr., en $A u t$., que dice era ya «de poco uso»..."; in Encina, Repres. del Amor, v. 284: "¿Tu quieres que llame al creco / o traya el físico luego...?»; v. 300: "Más quellotra un palaciego / que no físico ni crego...", donde non s'intende l'affermazione del "poco uso". "Fisonomía [filosomia, Celestina...]"; anche in Quirós (Canc. cast. s. XV,n. 564, v. 35): "Segun las filusumias...».

FLACO. Aggiungi Berceo, FLAQUIELLO 'debole, stanco', Mil. 29 I.

FLECO. "Flocar, ant. 'tirar, lanzar (algo a alguien)' (en 
Alonso de Solaya, $3^{\circ}$ cuarto del S. XV...), tambiéll cat. flocar 'pegar', 'echar' (de uso no general; Bulbena, Ag., Fabra; ya en Jaume Roig: "l'humit si $t$ toca / la mort te floca)...), explicable semánticamente por el significado de 'brizna, objeto despreciable' que tenía el lat. Floccus..."; FLOCAR e FROCAR ampiamente si documentano in egloghe e farse rinasc. nei sensi più vari; ad es., in Encina, Repclón, p. I28: "¡Oh, qué palo le froqué / en aquellos rabaciles»! Egl. de Antrucjo, p. 64: "y miefé, sus, a cantar, / y verás cómo le frocon (per altri ess. v. Gillet, III, nota a p. 3I6-7). Corominas s'accorda con Cejador nel riportare flocar a FLǑCCUS; Gillet, attraverso il dial. salm. riferisce frocar ad aflojar: "aflojar la cuerda (de un arco o una ballesta)", donde "soltar, descargar»; nell'es. di Encina il pastore si vanta di saper pizzicare bene il suo strumento musicale; frocar $=$ aflojar già in Lamano, p. 464.

FLUIR. "IN FLUJO [1605, Quijote; I618, Góngora...]"; in Herrera, III, 24, 5: "Con fiero influxo... / ... / el Cielo se llevó... / la luz mas pura d'Austria".

FOLLÓN. Aggiungi ENFELLONARSE 'infuriarsi', Berceo, S. Lor. 45. In "Berceo, Mil. 34", FELL $O N$ non significa "iracundo'", ma 'superbo': "con la qual [fionda di David, la Vergine] confondió al gigant tan fellón»; "iracundo'", invece, negli altri passi cit.; ad es. Mil. 56r: "el bispo... sannoso e irato... tornóse al conviento bravo e mui fellónn. cioè, '(giustamente) infuriato'.

FONETICO. "EPI FONEMA."; sta in Herrera, p. Ig6: "epifonema o aclamacion, que es cuando se sigue sentencia con admiración".

FONTA. Fr. honte: in Berceo, S. Lor. 39; $A$ FONTA, Mil. 344; FONTAR 'oltraggiare', ib. 909: "LLos malos que vinieron a fontar la tu ciella»; $A$ FONT $A D O$, ib. 383: "Tóvose la Gloriosa mucho por afontada»; ib. I54: «Por un ladrón non fuesse tal villa afontada»; S. D. 65I: "So de los enemigos de la fe afontadon.

FORMA. Aggiungi AFFORMADO, Berceo, Sacr. I65: tha cruz afformada». 
FORTUNA. "INFORTUNIO [I570, Mármol]》; forse anteriore in Herrera, el. II, 5, I60, giacché.v. II9 e I36: della cit. elegia sono riportati in $A$ p. I40: "Qu'en este m'infortunio i mal estraño".

FOSA. "fosal, ant. y arag., comp. cat. FOSSAR id..."; in Berceo, S. D. IIr: "Soterólo el fijo en el mismo fossar».

FRAGUA. Aggiungi FRAGUAMIENTO Berceo, 'edificio', Loor, 168.

FRANJA. "FIMBRIA.»; in Berceo, Loor. 2.

FRENTE. Aggiungi AFFRUENTO 'afflizione', Berceo, $S$. D. 4II: "So en fiero affruento con tal enfermedat».

FRIO. Corominas legge friura in Berceo, Mil. 6r3; Solalinde FRIDURA. "Restriar [Nebr.]"; aggiungi la forma RE$F R I A R$, in Encina, Égl. de Plác., p. I87 ("Ardo / de suerte que me refrion) e in Herrera, III, 39, 2 (H: "refriò - P: «refriar pudo el fuego ardiente»); Lamano, p. 603, cita il "refrán»: «En agosto refría el rostro».

FUENTE. Aggiungi la forma FONTE, in San Juan de la Cruz (vi D. Alonso, Poesía española, M. I950, p. 290.

FUERO. "Aforar 'otorgar fueros' [h. I29o...]"; A FORADO, in Berceo, Loor. I49, 'privilegiato'.

FUERTE. Aggiungi $A F O R C ̧ A R$ 'violentare', in Calila, p. I77: "commo la muger que se dió a su siervo dubdando e la aforçó» (in $C$ "la deshonró»). AFFORÇADO in Berceo, $S . M .48$, 'reso forte' («afforçado de la santa creençia e S. D. 744, 'valoroso' ( $\mathrm{E} 1$ Conçeio de Fita firme e afforf̧ado»); in questo stesso poema, 388, leggesi in $E$ e $V^{2}$ "esforfado», negli altri testi "DES FORZADO». Ancora da Berceo, ib. 225, aggiungi CONFUERTO. "Forsejudo»; ESFOR$C E J U D O$ in Encina, II Egl. en requesta, p. 73: "Enfinges de esforcejudo / adonde no es menester».

FULGOR. "FULGENTE [Gallegos, I626]"; frequente in Herrera (el. II, 3, 37; II, 52, I3; II, 8I, 2; el. II, I0, 6; el. I, 9, 7; I, 9, 5; ecc.). "RE FULGENTE [Oudin, no Covarr.; Aut. sin ejs.]»; per tali vocaboli basta, al solito, Herrera, $A$, p. I78, v. I0: "el variado monte refulgente». "FULGURAR [Villamediana, † I622]"; ancora Herrera. 
(I, 43, II; c. II, 5,45 , sólo in P). Il cantore di Luz conosce bene anche il verbo "Fulminar [Góngora, frecuente, y típico de su estilo... J" nelle forme (trasmesse allo stesso Góngora) FULMINANTE (c. II, I, I30: $H$ : "rayo resonante» - P: "braço fulminante») e FULMINADO (c. II, 3. I6: «i los fieros gigantes fulminados»); FULMINAR in Fray Luis de León; VII, 65: "menea fulminando el hierro insano"; XXXVI, p. I2I4: "Ni cuando sobre ti fulmina y truenan; chiara la fonte classica da Verg., Georg. I, 665, pp. I564: "Mas si fulmina de do el Cierzo espira" (lat. ofulminat").

FUNERAL. "FUNESTO [1605, Quijote...; falta todavía en Covatr...]"; in Herrera, $A$, p. 53, v. 30: "de funesto ciprés la triste frenten e in Fr. Luis de León, Del mundo y su vanidad (attribuita), v. I43.

FURIA. "FURIBUNDO [I605, Quijote]..."; in Fr. Luis de León, XI, 76, p. I450: "El furibundo Marte...».

FUSTE. Citazioni letterarie di FUSTA 'nave'. prima che in Cervantes, nei Cancioneros; ad es. "E las velas ya rompidas / e la fusta descosida" ( $J$. de Dueñas, La nao de $A$ mor, str. I3); "Del mesmo madero es nuestro nauio / que fueron las fustas de nuestros pasados" (G. Manrique, op. cit., p. 59); "Avnque mis fustas amarro (id.).

GAFA. " . $^{\mathrm{a}}$ doc.: 1570 , C. de las Casas... Covarr. (I6II)... Góngora...."; in Quirós, cit., p. 294: "Vn jubón le vi vestido, / rico, de brocado pelo, / y en medio, de brocadelo / vnas gatas esculpidas, / con vnas letras bruñidas / que de muy lexo se vían...n.

GAFO. "gafura y gafez 'ponzoña', 'sabandijas', 'reptiles'... ga/ura 'ponzoña' en el ast. de Colunga...n; GAHURRA 'scherno', in Berceo, Duelo I77: "escarnio e gahurras". V. hurriallá qui alla voce ARRE.

GALA. "GALANIA, ant. [h. I570, Ambr. de Morales]"; in Encina, II Egl. en requesta, p. 66: "(Escudero) Guarde Dios tu galania». Aggiungi GALISTO, ib., p. 82: "si me visto / los mis hatos domingueros / y si mudo aquestos cueros, / que te mando mal galistor. 
GALEA. Aggiungi GALEADOR 'imbroglione, impostore,' in Berceo, Duelo I67 e Mil. 687.

GALLARDO. Aggiungi GAYÓN 'elegantone, ruffiano', in Encina, Egl. de Plác., p. I79: "¿Cuál de aquellos, Gil Cestero, / era, soncas, el gayón?»; riportabile a galhon occ. Nella voce GAJO trovasi l'omonimo "gayón especie de forcáu de palo...».

GALLO. "Gallocresta [APal...: «duratio, yerva que dizen gallocrestan; Nebr.], del lat. GALLICRISTA, con influjo fonético de gallon; GALLICRESTA in Encina, Égl. en requesta, p. 69: "gallicresia y arvejones".

GENTE. Tutta una serie di vocaboli (gendarme e composti, genealogia e comp., genearca, geneático) è rimandata a questa voce, ma è stata dimenticata. Aggiungi GENTILHOM BRE, in Encina, II Égl. en requesta, p. 86 ("Por $\mathrm{mi}$ vida, Mingo hermano, / que estás así gentilhombre»; Égl. de Plác., p. I93 ("Decínoslo, gentilhombre»); Repres. del Amor, página 394 («Es un galán gentilhombre»).

GIRO. "La ac. etimológica 'circulo' se halla no sólo en APal., sino tambiéll en Barén de Soto (I643), y en Suárez. de Figueroa (I6I5)"; è preferenza herreriana dell'ultimo testo $P$ : norbe del fuego" $(H)$ - "giro d'el fuego" ( $P, \mathrm{c}$. II, I, II3); "alto polo" (B) - "giro ecelso" ( $P$, el. I, 9, 40); quindi passa a Góngora. Aggiungi GIRADORA, in Quirós (Canc. cast., cit. n. 564, t. II, p. 294): "Illeuaua vna capa encima / de giradoras chapada".

GITANO. Manca.

GLADIO. "Alex... Apol..."; in Berceo, Duelo 44: "Sin gladio $\mathrm{y} \sin$ lanza".

GLOBO. «del lat. globus... $I^{\text {a }}$ doc.: lo castellaniza ya APal..., pero no parece tuviera uso en castellano por entonces; $\mathrm{I}_{6 \mathrm{r}} \mathrm{7}$, Oudin; I6r5, Quijote... Con razón observa Capmany a fines del S. XVIII... que es afrancesado decir el globo por el globo terráqueo, como hacían y hacen algunos»; naturalmente il vocabolo non può mancare in Herrera: c. I, 4, 94: "de vos el fixo globo, i el tendido / umor, i el vago cerco se sustenta, / i el ardor de las llamas inquieton, che sono i quattro ele- 
menti, come è spiegato più avanti: a tierra i agua, '1 aire, 'l puro fuegoll; globo passa a Góngora, pur con significato generico.

GRADO I. Si noti in Herrera il significato del vocabolo nella variante: "mayor alteza» $(B)$ - «ublime grado $(P, c . I$, 4, 37).

GRADO II. ugradesçe de (agradesçedero) en el Rim. de Palacion; AGRADECEDERO, in Calila, p. 49.

GREN $A$. "U doc.: ...I586, Góngora....; in Encina, II Égl. en requesta, p. 74: ¿De qué te perturbas, di? / Si nunca medre tu greñan.

GRII,LO. Aggiungi ENGRILLARSE 'insuperbire', in Encina, II Egl. en requesta, p. 8 I (v. qui DUEÑO).

GRIMA. " $I^{2}$ doc.: APal... El vocablo vuelve a aparecer en la traducción del Cartujano (a. I502...), en Pérez de Hita...»; sta prima in Encina, Egl. de Carnal, p. 50: «de cúido, grima y cordojon.

GRITAR. Aggiungi GRITILLO, in Encina (v. qui ARRE). GUARDAR. "guardador"; $A G U A R D A D O R$ 'persona accompagnatrice', in Berceo, S. D. 532: "Ixo end el Obispo, e sus aguardadores".

GUIJA I. "GUIJO [Acad. ya I8I7]"; in Encina, Repr. del Amor, v. II6 (v. qui HERIR).

GUIÑAR. Aggiungi la forma GUIÑEAR, in Fr. L. de León, Job, XV, p. 995: «... ¿Qué guiñea, / qué amenaza tu rostro, frente y sienes?».

HABER. Aggiungi il sost. AVENTE 'possessore', in Berceo, Mil. 698: "Los pueblos de la villa... / ... / ...daban a non aventes / sus carnes...».

HALAREA. 'Comandamento, incitamento', in Berceo, S. D. 735: "A él serujá la ujlla e todas sus aldeas, / la su mano besauan, dél prendián halareas" (v. ¡HALA!).

HÁLITO. "Exalar [I590, J. de Acosta...]»; ESALAR in Herrera, frequentissimo: "i de la pluvia esala fuego ardiente» (el. II. II, I43, già in $B$ ); "II aunque las llamas todas esalasse / de su ahumada cumbre Tifoéō" (el. I, 7, I3); "el pecho esala todo" (II, I3, 87); "De mi pecho esaló un Ve- 
suvio ardiente" (II, 24, 9); "esala el fuego"(III, I4, Io); "esaló mayor llama" (III, 38, II). In Góngora ha altro significato. "ESALACI $O N$ ", in Herrera, el. II, 3, 45.

HARTO. Aggiungi il p. pr FARSIDO, in Berceo, Sacr. $25 \mathrm{I}$. "De buena farina es toda bien farsida".

HEBILLA. Aggiungi $A F I B L A R$, in Berceo, S. D. 156 : "Afiblóse el manto".

HELIO. Per il vocabolo e suoi composti si rimanda a SOL. Tuttavia desidero annotare il raro cultismo HELIOCRISO, in Herrera, $A$, p. 58, v. I3: "Nacera siempre eterno el amaranto, / Narcisso i eliocriso deleitoson; la fonte classica è Plinio, l'italiana il Molza e Annibal Caro; è l'elichrysim orientale, in it. perpetuino, in sp. perpetua amarilla (v. Dizionario della lingua italiana di Tommaseo e Bellini).

HERALDO. Su "faraute" e "filibustero" giova di riflesso la ricerca del giusto etimo di farabutto, compiuta da Gianfranco Contini, Un presunto ispanismo italiano da eliminare, in Estudios dedicados a Menéndez Pidal, t. II, pp. I49I62, M. I95I.

HERIR. Aggiungi il dial. FRETIR, in Encina, Repres. del Amor, v. I66: «i guarte que, si me descingo / mi hondijo, / fretirt'he en la cholla un guijon; omonimo di fretir, alla v. FREIR.

HERMANO. "GERMAN $1 A$.... 'rufianesca, hampa' [r609, J. Hidalgo]"; retrodatare ai primi decenni del S. XVI, in Rodrigo de Reinosa e Feliciano de Silva (I534), secondo Menéndez Pelayo, Origenes, V, pp. 20 e 74.

HEROE. "Heroico [APal...; Com. Griego; su uso se generalizó antes que el de héroe, a juzgar por el hecho de que es más frecuente que éste en Góngora]”; EROICO è vocabolo fondamentale del lessico herreriano, nel quale è dato meglio misurare l'accennata frequenza.

HILO. Aggiungi REHILAR 'brillare degli occhi', in Encina, II Égl. en requesta, p. 78: "Xa se te rehila el ojo. / Ya de ti no tengo enojo". "HILA... 'hilera' ant. (Ercilla, Inca Garc., Mariana...); 'hebra de lienzo...', comúnmente en 
plural [Quijote...]"; in Encina, Repelón, p. I22: "No habria hilas en ellos [gli studenti] / si enel campo los tuviesell.

HINCAR. "Ahincar [af- 125I, Calila...]; ahincado..."; AFINCADO in Berceo, Mil. 206: "Fo bien de cada parte afincada la cosan; anche $A F I C A M I E N T O$ 'pena, angoscia', S. M. 82.

HINCHAR. Aggiungi FINCHIDOR, in Villasandino (Respuesta de Fr. de Baena, Canc. cast. s. XV, t. II p. 369): "Sy entrades en mi huerto / fynchidor de las privadas, / yo vos porné almohadas / que vos assyenten en cierto" $H I P A L A G E$. In Herrera, $A$, p. 304: «metonimia... los oradores la nombraron ipálage, que significa trasmudación...". HIPERTESIS. In Herrera (ipértesis), $A$.

HONDO. Aggiungere la forma $J O N D O$ e ricordarue il significato in cantc jondo, che tanta fortuna, anche letteraria, ha avuto nel nostro secolo.

HORNO. "FORNICARIO [Nebr.]"; in Berceo, Mil. 78: tanto pudió bullir el sotil aversario, / que corrompió al monge, fizo lo fornicarion.

HORRO. Aggiungi AFFORRAGE, in Villasandino, cit., p. 365: "Los que están / con sant Bulán / e buscan otro afforrage...",

HORROR. "HORRÍSONO, de horrisōnus...»; è cultismo herreriano: es., "orrisono estampido" ( $P$, el. II, II, I53).

HUCIA. Nei deriv. con des- aggiungi DES FUZIARSE, Calila, pp. I59, 210, 218, 252, quindi si documenti, oltre al genere pronominale, la forma $D E S A F U Z I A R$, che ise hace frecuente desde el S. XIV", di fronte a DES FIUZAR, "la forma corriente desde Berceo hasta Juan Manuel" ("desafiuçar, Alex." e "desfeiiçar del ms. P» ritenuta "más auténtica"), "enfinzzar (Acad.)"; ENFIUZARSE, Calila, pp. 420 e 460; $A F E U Z I A R S E$, ib., p. 274; FYUZARSE, ib., página 344; DEFEUZADO in Berceo, Duelo 5; DESFIUZIARSE, Calila, p. 330.

HUIR. "FUGAZ [x640, Saavedra]...»; in Fr. Luis de León, $\mathrm{X}, 30, \mathrm{p}$. I448; $\mathrm{XI}, 30$ : "cuanto es fugaz y vano aquel contenton. 
HUMANO. «humanal... envejece después del S. XV... [Berceo, todavía Nebr.]"; ancora in Fr. Luis de León, XIX, 35, p. I467: "del linaje humanal fiel abogada" (il verso ha una sua arcaica solennitá, nello stile di Berceo, e v. qui ABOGADO).

¡HUY!. «( ${ }^{\mathrm{a}}$ doc.: h. I840, Hartzenbusch (en Pagès); Acad. I884... Creo haberla leído en el Quijote»; leggesi in Encina nei versi rotti del villancico che chiude l'Egl. de Navidad: «jHuy hó!... ¡Huy há, huy hó!», nel ritornello e nelle sei strofe.

IDIOMA. "IDIOTISMO [Aut.]... 'locución propia de una lengua'»; in Herrera, $A$, p. 307: "muerta, cansada»-quiere dezir afligida i fatigada que es idiotismo i figura auxésis que es amplificacion, incremento, esageración i crecimicnto en nuestra lengua...; contraria desta figura es la que en Griego se nombra miosis o tapinosis, i en latin imminucion o desminucion o estenuacion... es tropo con que se deprimen i umillan las cosas mas de lo que es juston.

IGUAL. Annota il senso lat. 'giusto' (aequus): Fr. Luis de León, II, 32, p. I432: "En pueblo inculto y duro / induce poderoso, igual costumbre». Aggiungi $E G U A D O$ 'nel mezzo della vita, adulto', in Berceo, Sign. 24: "Quantos nunca murieron en qualquiera edat / ninnos o eguados o en grant vegedat». "EQUIVOCO...», applicato da Herrera ( $A$, página 254) al verso leonino.

IJADA. Aggiungi TRASIJAR, ecc.

IMAGEN. Aggiungi IMAGINANZA, in Villasandino (op. cit., p, 379): «ymaginança».

INCLITO. " "I doc.: APal... Falta en Nebr., pero figura un par de veces en el Quijote, está en Mariana, Covarr., etc.»; frequentissimo in Herrera, es.: $B, \mathrm{c} . \mathrm{V}, \mathrm{I} 56 ; \mathrm{I}, 57, \mathrm{I} 4$; el. I, 7, 70; II, 40, I3; el. III, I, I65; III, I3, 8; c. III, 3, 23; come sost., c. II, 6, I79; trovasi in Góngora; ma già in poeti del 400: in Juan Poeta ynclito e in Juan de Mena ynclita (v. Rosa Lida, pp. 278 e 255); C. de las Casas traduce l'it. inclito con uillustre, famoso»; in Fr. Luis de León, Quien viere, v. 37, p. I494: "inclitas hazañas». 
ITEM. Manca. Aggiungi la forma ITEN, frequente nei cancioneros: es., Qnirós, cit., t. II, p. 298: "Itén más, que amor alcança...».

JARA. Aggiungi la forma JARIN (xara nel testo $B$ ), dello stesso.genere e signif., in Calila, p. 430.

JILGUERO. "El adjetivo silgo... aporta una buena confirmación del étimo aquí aceptado, y xerguerito en una Farsa del S. XVI (Fcha.) es derivado de jerga, sinónimo de sirgon; in Encina, Égl. en requesta, p. 69, la forma JERGUERITO. JUEGO. "JUGLERIA, J. Ruiz"; in Calila, p. I: "et plazíales más de aquello que de ninguna jugleria nin de otro plazern.

JUNTO. "COYUNTURA [... "coiuntura: junctura; conjunctio; c. de dedos: codillus», Nebr.]...n; aggiungi il senso traslato di 'opportunità, momento favorevole', ad es., in Herrera ("siendo justo perder tal coyuntura", el. I, 4, 2I, già in $B$ ) e in Góngora ("gran coinnctiva a de ofrecerse...").

Oreste Macrí.

Universita di Firenze. 